\title{
Intestinal transport mechanism and in vivo anticancer efficacy of a solid oral formulation incorporating an ion-pairing complex of pemetrexed with deoxycholic acid derivative
}

This article was published in the following Dove Press journal:

International Journal of Nanomedicine

Rudra Pangeni ${ }^{1, *}$

Saurav Kumar Jha ${ }^{1, *}$

Ruby Maharjan ${ }^{2}$

Jeong Uk Choi ${ }^{2}$

Kwan-Young Chang ${ }^{3}$

Young Kweon $\mathrm{Choi}^{3}$

Youngro Byun ${ }^{4}$

Jin Woo Park'

'Department of Pharmacy, College of Pharmacy and Natural Medicine Research Institute, Mokpo National University, Jeonnam 58554, Republic of Korea; ${ }^{2}$ Research Institute of Pharmaceutical Sciences, College of Pharmacy, Seoul National University, Seoul 08826, Republic of Korea; ${ }^{3}$ Global R\&D

Center, Icure BNP, Seoul 06170, Republic of Korea; ${ }^{4}$ Department of Molecular Medicine and Biopharmaceutical Science, Graduate

School of Convergence Science and

Technology, College of Pharmacy, Seoul

National University, Seoul 08826, Republic of Korea

*These authors contributed equally to this work

Correspondence: Youngro Byun Department of Molecular Medicine and Biopharmaceutical Science, Graduate School of Convergence Science and Technology, College of Pharmacy, Seoul National University, I Gwanak-ro, Seoul 08826, Republic of Korea

Tel +8228807866

Fax +8228727864

Email yrbyun@snu.ac.kr

Jin Woo Park

College of Pharmacy and Natural Medicine Research Institute, Mokpo National

University, 1666 Youngsan-ro, Muan-gun, Jeonnam 58554, Republic of Korea

Tel +82 6I 4502704

Fax +82 6I 4502689

Email jwpark@mokpo.ac.kr
Objective: The rational combination of immunotherapy with standard chemotherapy shows synergistic clinical activities in cancer treatment. In the present study, an oral powder formulation of pemetrexed (PMX) was developed to enhance intestinal membrane permeability and investigate its application in metronomic chemotherapy in combination with immunotherapy.

Methods: PMX was ionically complexed with a bile acid derivative ( $\mathrm{N}^{\alpha}$-deoxycholyl-Llysyl-methylester; DCK) as a permeation enhancer and mixed with dispersing agents, such as poloxamer 188 (P188) and Labrasol, to form an amorphous oral powder formulation of PMX/DCK (PMX/DCK-OP).

Results: The apparent permeability $\left(\mathrm{P}_{\mathrm{app}}\right)$ of PMX/DCK-OP across a Caco- 2 cell monolayer was 2.46- and 8.26-fold greater than that of PMX/DCK and free PMX, respectively, which may have been due to the specific interaction of DCK with bile acid transporters, as well as the alteration of membrane fluidity due to Labrasol and P188. Furthermore, inhibition of bile acid transporters by actinomycin D in Caco-2 cell monolayers decreased the $\mathrm{P}_{\text {app }}$ of PMX/ DCK-OP by $75.4 \%$, suggesting a predominant role of bile acid transporters in the intestinal absorption of PMX/DCK-OP. In addition, caveola/lipid raft-dependent endocytosis, macropinocytosis, passive diffusion, and paracellular transport mechanisms significantly influenced the permeation of PMX/DCK-OP through the intestinal membrane. Therefore, the oral bioavailability of PMX/DCK-OP in rats was $19.8 \% \pm 6.93 \%$, which was $294 \%$ higher than that of oral PMX. Moreover, an in vivo anticancer efficacy study in B16F10 cell-bearing mice treated with a combination of oral PMX/DCK-OP and intraperitoneal anti-PD1 exhibited significant suppression of tumor growth, and the tumor volume was maximally inhibited by 2.03- and 3.16-fold compared to the oral PMX/DCK-OP and control groups, respectively. Conclusion: These findings indicated the therapeutic potential of a combination of low-dose oral chemotherapy and immunotherapy for synergistic anticancer efficacy.

Keywords: pemetrexed, bile acid derivative, oral powder formulation, intestinal membrane permeability, oral delivery, oral metronomic chemotherapy

\section{Introduction}

Intravenous administration of the maximum tolerated dose of an antineoplastic chemotherapeutic agent has been widely accepted as a clinical strategy to obtain complete remission or cure of cancer. However, this strategy is associated with the development of chemoresistance and both long- and short-term toxicity, including 
myelosuppression, neutropenia, thrombocytopenia, and gastrointestinal dysfunction, along with the depletion of both innate and adaptive defense mechanisms. To address these issues related to conventional chemotherapy, a novel approach involving frequent low-dose oral administration, also known as metronomic chemotherapy, has been suggested, which maintains uniform serum levels of drugs, overcomes drug resistance by targeting the tumor vasculature, and avoids toxic effects. ${ }^{1}$ Moreover, metronomic chemotherapy inhibits tumor angiogenesis, promotes activation of innate and adaptive immunity, and induces tumor dormancy. $^{2}$ However, metronomic scheduling for improved patient quality of life can be preferentially achieved by oral drug administration. In addition, oral administration is considered one of the safest and most convenient routes to maintaining prolonged exposure of cancerous cells to chemotherapeutic agents. However, oral delivery of these anticancer drugs is a major challenge due to their physicochemical properties, variable rate of degradation in gastrointestinal fluid, and high drug efflux through P-glycoprotein (P-gp). ${ }^{3,4}$ Furthermore, recent studies suggested the use of low-dose chemotherapeutics in combination with immunotherapy to overcome the immunosuppression induced by cancer, intensify host immunity to destroy tumor cells, produce highly active tumor-specific $\mathrm{T}$ cells, and synergistically optimize antitumor effects. $^{5,6}$ Therefore, the rational combination of immunotherapy with oral low-dose chemotherapy is expected to control tumor growth and address the immunosuppressive tumor environment.

Pemetrexed (N-[4-[2-(2-amino-3,4-dihydro-4-oxo-7Hpyrrolo[2,3-d] pyrimidin-5-yl)-ethyl]-benzoyl]-L-glutamic acid; PMX) is a novel multi-targeted antineoplastic agent that disrupts metabolic processes related to the de novo biosynthesis of thymidine and purine nucleotides. Clinically, PMX in combination with cisplatin has been approved by the US Food and Drug Administration as the first-line treatment of patients with non-squamous and non-small cell lung cancer (NSCLC), as well as to treat relapsed or refractory NLCLC after chemotherapy with platinum-based regimens. Moreover, treatment of advanced NSCLC with a combination of PMX and immunotherapeutic agents, such as bevacizumab, has been recommended as maintenance therapy, where both drugs are highly effective, well tolerated, and exhibit low toxicity. $^{7}$ PMX is a polar-charged compound with a relatively small distribution volume and rapid clearance.
Moreover, PMX exhibits low oral bioavailability, which may be due to its poor intestinal absorption. ${ }^{8,9}$

To overcome these obstacles, various approaches, including coadministration of therapeutic agents with functional excipients (P-gp inhibitors) or use of permeation enhancers, have been developed. ${ }^{10-12}$ In addition, polymeric or lipid-based drug carriers, such as polymeric nanoparticles, polymeric micelles, microemulsions, selfemulsifying drug delivery systems, lipid-drug conjugates, and nanocrystals have been used to improve the oral bioavailability of drugs. ${ }^{13,14}$

There is increasing interest in the design of potential prodrug targets for carrier-mediated uptake via bile acid transporters to improve intestinal drug absorption. ${ }^{15,16}$ The prodrug approach includes chemical modification of drugs to improve their charge and lipophilicity, to in turn promote intestinal permeation. Prodrugs targeting endogenous transporters present on enterocytes can facilitate site-specific transport and represent a novel strategy for intestinal delivery of drugs with very low oral bioavailability. ${ }^{15}$ Various approaches to exploit the prodrug concept have already been applied to increase the central nervous system (CNS) delivery of several drugs. Bonina et al reported greater reserpine-induced hypolocomotion effects in rats when composites were linked via the C-3 glucose position. ${ }^{17}$ Bilsky et al demonstrated increased uptake of opioid peptide agonist through the blood-brain barrier (BBB) after conjugation with D-glucose. ${ }^{18}$ In both cases, the prodrug was targeted to glucose transporter (GLUT) expressed in the BBB. Similarly, terminal ileum of the intestine is the major site of bile acid reabsorption in humans, and bile acid is actively transported to the terminal ileum by the wellcharacterized apical sodium-dependent bile acid transporter (ASBT). ${ }^{19}$ The use of prodrugs to target ASBT and increase intestinal drug permeability is widely practiced. For example, acyclovir showed an unambiguous increase in oral bioavailability via a prodrug targeting ASBT, ie, acyclovir valylchenodeoxycholate. $^{20}$

In our previous studies, we prepared an ionic complex of hydrophilic drugs with a bile acid derivative $\left(\mathrm{N}^{\alpha}\right.$-deoxycholylL-lysyl-methylester; DCK) as a permeation enhancer. ${ }^{16,21,22}$ DCK was prepared by chemical linkage of deoxycholic acid (DOCA) with positively charged L-lysine, which showed increased intestinal permeability of hydrophilic drugs, such as bisphosphonates, insulin, and oxaliplatin. The ion-paired complex is known to be less stable than the chemically conjugated prodrug form, and a major concern when utilizing the ion-pairing approach is the possibility of premature complex 
dissociation in the biological fluids prior to absorption. ${ }^{23}$ However, chemical modification by attaching a functional molecule to the drug may result in loss of biological activity; new tests to assess safety and efficacy are required in accordance with the development of novel drug therapies. ${ }^{24}$ Furthermore, the ion-paired DCK can more effectively deliver specific target molecules through the intestinal membrane via ASBT-mediated endocytosis and/or an increase in concentration gradients through the intestinal membrane compared to other non-specific and physically mixed absorption enhancers, such as surfactants. ${ }^{25}$ Recently, we also prepared an ion-pairing complex of PMX and DCK in combination with dispersants, such as 2-hydroxypropyl- $\beta$-cyclodextrin (HP- $\beta$-CD) and poloxamer 188 (P188), forming the HP- $\beta-C D / P M X /$ DCK/P188 complex. Furthermore, we incorporated the ionpairing complex into the inner aqueous phase of multiple water-in-oil-in-water nanoemulsions in a supersaturated state. Both the HP- $\beta-C D / P M X / D C K / P 188$ complex and its nanoemulsion showed significant increases in the oral bioavailability of PMX, which may have been due to increased lipophilicity of the complex caused by DCK, disruption of the intestinal barrier function by DOCA, and surfactant-induced membrane fluidity. However, incorporation of HP- $\beta-\mathrm{CD} /$ PMX/DCK/P188 complex into the aqueous phase of the nanoemulsions resulted in stability problems during longterm storage. The oral powder formulation of PMX effectively enhanced oral bioavailability and drug stability.

The main objective of the present study was to design an oral powder formulation of PMX for application in metronomic chemotherapy, as well as for combination use with immunotherapy. PMX was ionically complexed with DCK in combination with a dispersing agent, such as P188 or Labrasol, forming an oral powder formulation of PMX/DCK (PMX/DCK-OP). This can be effectively absorbed across the intestinal membrane due to increased lipophilicity of PMX achieved by ion-pairing complex with DCK and interaction with bile acid transporters. In addition, stable formation of micelles in the aqueous solution, dispersant activity of P188, opening of tight junctions (TJs), and surfactant activity of Labrasol can also enhance the intestinal membrane permeability of PMX. Therefore, to confirm the increase in oral absorption of PMX after preparation of an oral powder formulation, we determined the artificial membrane and Caco-2 cell monolayer permeability of PMX/DCK and PMX/DCK-OP. The intestinal transport pathways of PMX/DCK-OP through a Caco-2 cell monolayer were then assessed using specific pharmacological inhibitors. Finally, the oral bioavailability of
PMX/DCK-OP in rats, and its antitumor efficacy in combination with intraperitoneal anti-PD1 antibody in tumorbearing mice, was evaluated.

\section{Materials and methods Materials}

The PMX disodium hemipentahydrate was obtained from Shipla Medicare (Karnataka, India). Caprylocaproyl macrogol-8-glyceride (Labrasol) was provided by Gattefossé (Saint Priest, France). Polyoxyethylene (160) polyoxypropylene (30) glycol (P188) was purchased from BASF (Ludwigshafen, Germany). Chlorpromazine, methyl- $\beta$-cyclodextrin (M $\beta C D)$, brefeldin A, genistein, actinomycin D (Act D), cyclosporine A, ethylene glycol-bis-( $\beta$-aminoethyl ether)-N,N,N',N'-tetraacetic acid (EGTA), DOCA, and 4-[N-(2,4-diamino-6-pterinidinylmethyl)-N-methylamino]benzoic acid hemihydrochloride hydrate (DAMPA) were obtained from Sigma-Aldrich Inc. (St. Louis, MO). Anti-mouse PD-1 antibody (anti-PD1) was purchased from Bio X Cell (West Lebanon, NH). Solvents for high-performance liquid chromatography (HPLC) were obtained from Merck Millipore (Billerica, MA) and Thermo Fisher Scientific Inc. (Waltham, MA).

\section{Animals}

Sprague-Dawley rats (males, 6-7 weeks old, 200-250 g) and BALB/c mice (females, 6-7 weeks old, 20-25 g) were provided by Orient Bio (Gwangju, Republic of Korea). The animals were housed under environmentally controlled conditions of temperature $\left(23{ }^{\circ} \mathrm{C} \pm 2{ }^{\circ} \mathrm{C}\right)$, relative humidity $(55 \% \pm 10 \%)$, and light $(12 / 12 \mathrm{hr}$ light/dark cycle). The animals were fed a standard laboratory diet (Nestlé Purina, St. Louis, MO) and ion-sterilized tap water. Ethical approval for this study was obtained from the Institutional Animal Care and Use Committee (IACUC) of Mokpo National University (Jeonnam, Republic of Korea). All animal experiments were performed in accordance with the NIH Guidelines for the Care and Use of Laboratory Animals and the guidelines of the IACUC.

\section{Preparation and characterization of PMX/ DCK-OP}

We synthesized DCK as an oral permeation enhancer, as described previously. ${ }^{16}$ Briefly, $\mathrm{H}-\mathrm{Lys}(\mathrm{Boc})-\mathrm{OMe} \cdot \mathrm{HCl}$ $(20 \mathrm{~g})$ dissolved in $N$-methyl morpholine $(7.4 \mathrm{~mL})$ and ethyl chloroformate $(6.4 \mathrm{~mL})$ were mixed with DOCA $(26 \mathrm{~g})$ in tetrahydrofuran $(800 \mathrm{~mL})$ with continuous stirring for $30 \mathrm{mins}$, followed by $2 \mathrm{hr}$ reflux. The precipitates 
obtained after stirring of the reaction mixture overnight at room temperature were collected by filtration and the residual solvents in the precipitates were removed by evaporation. Purified Lys(Boc)DOCA was obtained by column chromatography (chloroform/methanol) of the dried precipitates, which was further dissolved in a mixture of acetyl chloride and methanol in an ice bath. After complete removal of solvent, the residue was dissolved in water and washed three times with chloroform. Then, the aqueous layer was collected and freeze-dried to obtain DCK powder. Next, ion-pairing complex of PMX with DCK (PMX/DCK) was prepared by dissolving $50 \mathrm{mg}$ of PMX in $10 \mathrm{~mL}$ of deionized water. Then, $10 \mathrm{~mL}$ of DCK solution in deionized water $(6.68 \mathrm{mg} / \mathrm{mL})$ was prepared and added dropwise into the PMX solution at a molar ratio of 1:1, with vortex mixing after each addition. The solution mixture of PMX and DCK was freeze-dried at $-70{ }^{\circ} \mathrm{C}$ to obtain PMX/DCK complex powder.

To enhance the intestinal membrane permeability of PMX, PMX/DCK-OP was prepared using the solid dispersion technique. Briefly, PMX (50 mg) was dissolved in P188 solution prepared by dissolving $500 \mathrm{mg}$ of P188 in $10 \mathrm{~mL}$ of deionized water containing $375 \mu \mathrm{L}$ of Labrasol. DCK solution was prepared in another tube by dissolving $66.8 \mathrm{mg}$ of DCK in $8 \mathrm{~mL}$ of deionized water. At a molar ratio of $1: 1$, DCK solution was added dropwise into the PMX solution with regular vortex mixing. In addition, $80 \mu \mathrm{L}$ of $\mathrm{NaOH}(1 \mathrm{M})$ was added with thorough mixing to maintain the $\mathrm{PMX} / \mathrm{DCK}$ mixture at $\mathrm{pH} 7$. The mixture was then freeze-dried at $-70{ }^{\circ} \mathrm{C}$ to completely remove the water and obtain PMX/DCK-OP in powder form. Separately, to explore the role of DCK in enhancing the intestinal membrane permeability of PMX, an oral powder formulation of PMX containing Labrasol and P188 (PMXOP) was prepared. Briefly, $50 \mathrm{mg}$ PMX was dissolved in $10 \mathrm{~mL}$ of $\mathrm{P} 188$ solution $(50 \mathrm{mg} / \mathrm{mL})$ containing $375 \mu \mathrm{L}$ of Labrasol. The mixture was then well vortexed for $5 \mathrm{~min}$ and freeze-dried at $-70{ }^{\circ} \mathrm{C}$ to completely remove water and obtain PMX-OP in powder form. Furthermore, to confirm micelle formation of PMX/DCK-OP in the aqueous phase, the powder formulation was dispersed in deionized water (1:20, w/v) and its droplet size, polydispersity index (PDI), and zeta potential were measured with a dynamic laser light scattering (DLS) analyzer (Zetasizer Nano ZS90; Malvern Instruments, Malvern UK) at $25^{\circ} \mathrm{C}$. The morphology and particle size of PMX/DCK-OP micelles were further determined using transmission electron microscopy (TEM). After negative staining of the
PMX/DCK-OP dispersed in deionized water $(1: 100, \mathrm{w} / \mathrm{v})$ with $2 \%(\mathrm{w} / \mathrm{v})$ phosphotungstic acid solution, the sample solution was dropped on a copper grid and examined using high-resolution transmission electron microscopy (HRTEM; JEM-200; JEOL, Tokyo, Japan).

\section{In vitro artificial intestinal membrane permeability}

The in vitro artificial membrane permeability of free PMX, PMX/DCK, PMX-OP, and PMX/DCK-OP was determined using the parallel artificial membrane permeability assay. Sample solution $(200 \mu \mathrm{L})$ at a concentration equivalent to $200 \mu \mathrm{g} / \mathrm{mL}$ of PMX was added to each well of the donor plates, which were prepared by dilution of free PMX, PMX/ DCK, PMX-OP, and PMX/DCK-OP with phosphate-buffered saline (PBS, $\mathrm{pH}$ 6.8). We added $300 \mu \mathrm{L}$ of PBS ( $\mathrm{pH}$ $6.8)$ to each well of the receptor plates. After $5 \mathrm{hr}$ incubation at room temperature, the plates were disassembled and samples were withdrawn from both the donor and acceptor plates. The concentration of PMX that permeated through the artificial membrane was measured by HPLC at $254 \mathrm{~nm}$ with a C18 column $(4.6 \times 250 \mathrm{~mm}, 5 \mu \mathrm{m}, 100 \AA)$ at $25{ }^{\circ} \mathrm{C}$. The mobile phase consisted of water ( $\mathrm{pH} 3.5$ adjusted with phosphoric acid)-acetonitrile $(80: 20, \mathrm{v} / \mathrm{v})$ and the flow rate was optimized to $1 \mathrm{~mL} / \mathrm{min}$.

The effective permeability $\left(\mathrm{P}_{\mathrm{e}}\right)$ of each sample was calculated using the formula:

$$
\mathrm{P}_{\mathrm{e}}=-\ln \left[1-\mathrm{C}_{\mathrm{R}}(\mathrm{t}) / \mathrm{C}_{\text {equilibrium }}\right] /\left[\mathrm{S} \times\left(1 / \mathrm{V}_{\mathrm{D}}+1 / \mathrm{V}_{\mathrm{R}}\right) \times \mathrm{t}\right]
$$

where $\mathrm{P}_{\mathrm{e}}$ is the permeability $(\mathrm{cm} / \mathrm{s}), \mathrm{S}$ is the effective filter area $\left(0.288 \mathrm{~cm}^{2}\right), \mathrm{V}_{\mathrm{D}}$ is the volume of the donor well $(0.2 \mathrm{~mL}), \mathrm{V}_{\mathrm{R}}$ is the volume of the receptor well $(0.3 \mathrm{~mL})$, $t$ is the total time of incubation in seconds, $C_{R}(t)$ denotes the concentration of the drug in the receptor well at time $t$, and $\mathrm{C}_{\text {equilibrium represents }}\left[\mathrm{C}_{\mathrm{D}}(\mathrm{t}) \times \mathrm{V}_{\mathrm{D}}+\mathrm{C}_{\mathrm{R}}(\mathrm{t}) \times \mathrm{V}_{\mathrm{R}}\right] /\left(\mathrm{V}_{\mathrm{D}}+\mathrm{V}_{\mathrm{R}}\right)$, where $C_{D}(t)$ denotes the concentration of the drug in the donor well at time $\mathrm{t}^{16}$

\section{In vitro permeability across a Caco-2 monolayer}

To determine the apparent permeability $\left(\mathrm{P}_{\mathrm{app}}\right)$ of $\mathrm{PMX}$, PMX/DCK, PMX-OP, and PMX/DCK-OP, Caco-2 cell monolayers were formed by seeding Caco-2 cells (ATCC $^{\circledR}$ HTB-37TM; American Type Culture Collection, Manassas, VA, USA), at a density of $1 \times 10^{5}$ cells/well, on polystyrene Transwell membranes in Dulbecco's modified 
Eagle's medium (DMEM) containing 10\% (v/v) fetal bovine serum (FBS) and 1\% penicillin/streptomycin, followed by culture for 14 to 16 days. Media in the apical and basolateral compartments were replaced every other day until the transepithelial electrical resistance (TEER) reached $>350 \Omega \cdot \mathrm{cm}^{2}$. To investigate transcellular and ASBT-mediated transport, the culture medium in both the apical and basolateral compartments was removed and replaced with prewarmed HBSS. HBSS in the apical compartment was then replaced with $0.1 \mathrm{~mL}$ of PMX, PMX/ DCK, PMX-OP, or PMX/DCK-OP diluted in HBSS (equivalent to $100 \mu \mathrm{g} / \mathrm{mL}$ PMX), and that in the basolateral compartment was replaced with $0.6 \mathrm{~mL}$ of fresh HBSS followed by incubation of the plates at $37{ }^{\circ} \mathrm{C}$. Samples $(0.1 \mathrm{~mL})$ from each basolateral compartment were withdrawn at predetermined time points of $0,0.5$, $1,2,3,4$, and $5 \mathrm{hrs}$ and replaced with the same volume of fresh HBSS. The collected samples were then filtered through polyvinylidene difluoride (PVDF) membrane filters $(0.45 \mu \mathrm{m})$, and the concentrations of PMX in the samples were determined by HPLC with a UV detector at $254 \mathrm{~nm}$, as described above.

The $\mathrm{P}_{\mathrm{app}}$ for PMX in various formulations was calculated as follows:

$$
\mathrm{P}_{\mathrm{app}}=\mathrm{dQ} / \mathrm{dt} \times 1 /\left(\mathrm{S} \times \mathrm{C}_{\mathrm{i}}\right)
$$

where $\mathrm{dQ} / \mathrm{dt}$ represents the linear appearance rate of mass on the basolateral side $(\mu \mathrm{g} / \mathrm{s}), \mathrm{S}$ indicates the surface area of the monolayer $\left(\mathrm{cm}^{2}\right)$, and $\mathrm{C}_{\mathrm{i}}$ is the initial concentration of PMX on the apical side $(\mu \mathrm{g} / \mathrm{mL}){ }^{26}$

\section{Intestinal transport mechanism of PMX/ DCK-OP}

Next, we examined the role of ASBT in the permeation of PMX/ DCK-OP, as well as the endocytosis pathways of PMX/DCKOP micelles in the aqueous phase. Before performing the intestinal transport study, to determine the appropriate Act D concentration, the in vitro cytotoxicity assay results of Act D using Caco-2 cells were evaluated using the Cell Counting Kit-8 (CCK-8; Dojindo Molecular Technologies, Rockville, MD, USA). Caco- 2 cells were seeded at a density of $1 \times 10^{4}$ cells/ well in $200 \mu \mathrm{L}$ DMEM with $10 \%$ FBS in 96-well plates and cultured for $24 \mathrm{~h}$. The cells were then treated with serially diluted sample solutions of 1, 2, 3.2, and $6 \mu \mathrm{M}$ Act D in HBSS for $5 \mathrm{hrs}$. To evaluate the cytotoxic effect of Act $D$ on the Caco- 2 cells, a 10- $\mu$ L WST-8 (2-(2-methoxy-4-nitrophenyl)-3-(4-nitrophenyl)5-(2,4-disulfophenyl)-2H-tetrazolium monosodium salt) solution was added to each well and incubated for $1 \mathrm{hr}$. The absorbance was then measured using a microplate reader (Multimode Plate Reader; PerkinElmer Inc., Waltham, MA, USA) at $450 \mathrm{~nm}$. The results obtained for the treated cells are expressed as the percentage of viable cells compared to untreated cells. After determining the Act $\mathrm{D}$ concentration showing no toxic effect, Caco- 2 cells were cultured on Transwell membranes and the medium in the apical and basolateral compartments was replaced every other day until the TEER value of the Caco-2 cell monolayer reached $>350 \Omega \cdot \mathrm{cm}^{2}$, to establish the in vitro transcellular model. To investigate the possible transcellular pathways of PMX/DCK-OP transport, $0.1 \mathrm{~mL}$ of HBSS containing inhibitors of the specific cellular uptake pathway (Table 1) and $0.6 \mathrm{~mL}$ of HBSS were then added to the apical and basolateral compartments of each well, respectively. After preincubation at $37^{\circ} \mathrm{C}$ for $30 \mathrm{mins}$, the solution in the apical compartment was replaced with $0.1 \mathrm{~mL}$ of PMX/DCK-OP diluted in HBSS (equivalent to $100 \mu \mathrm{g} / \mathrm{mL}$ PMX) with corresponding inhibitors, and that in the basolateral compartment was replaced with $0.6 \mathrm{~mL}$ of fresh HBSS followed by incubation at $37^{\circ} \mathrm{C}$. Then, $200 \mu \mathrm{L}$ of sample solution was withdrawn at $0,0.5$, $1,2,3,4$, and $5 \mathrm{hrs}$ from the basolateral compartment of each well and replaced with the same volume of fresh HBSS. ${ }^{26,27}$

In addition, to evaluate the effects of $\mathrm{TJ}$ opening on the transport of $\mathrm{PMX} / \mathrm{DCK}-\mathrm{OP}$, the extracellular $\mathrm{Ca}^{+2}$

Table I Inhibitors with different functions used in the transport study and their concentrations

\begin{tabular}{|l|l|l|}
\hline Inhibitors & Concentrations & Functions \\
\hline Act D & $3.2 \mu \mathrm{M}$ & Inhibitor of ASBT-mediated transport \\
Chlorpromazine & $32 \mu \mathrm{M}$ & Inhibitor of clathrin-mediated endocytosis \\
$\mathrm{M} \beta C D$ & $10 \mathrm{mM}$ & Inhibitor of caveola/lipid raft-mediated endocytosis (cholesterol depletion agent) \\
Genistein & $0.1 \mathrm{mM}$ & Inhibitor of caveola/lipid raft-mediated endocytosis (broad inhibitor of protein tyrosine kinase) \\
Amiloride & $0.1 \mathrm{mM}$ & Inhibitor of macropinocytosis \\
Cyclosporine A & $10 \mathrm{mM}$ & Inhibitor of P-gP mediated efflux \\
Brefeldin A & $90 \mu \mathrm{M}$ & Inhibitor of ER/Golgi pathway \\
\hline
\end{tabular}

Abbreviations: Act $D$, actinomycin $D ; M \beta C D$, methyl- $\beta$-cyclodextrin; ASBT, apical sodium-dependent bile acid transporter; P-gp, P-glycoprotein; ER, endoplasmic reticulum. 
chelating agent, EGTA, was applied to reversibly open the intracellular TJs in the Caco-2 cell monolayer. Moreover, to examine the effects of EGTA in combination with all inhibitors except cyclosporine A on the transport of PMX/ DCK-OP, a Caco-2 cell monolayer with TEER value $>350 \Omega \cdot \mathrm{cm}^{2}$ was treated with $0.1 \mathrm{~mL}$ of $\mathrm{HBSS}\left(\mathrm{Ca}^{+2}-\right.$ free medium) containing $2.5 \mathrm{mM}$ EGTA with or without all inhibitors except cyclosporine A. After filling the basolateral side with $0.6 \mathrm{~mL}$ of $\mathrm{HBSS}$ ( $\mathrm{Ca}^{+2}$-free medium), the Transwell membranes were preincubated at $37{ }^{\circ} \mathrm{C}$ for 45 mins and the integrity of the cell monolayer was monitored by measuring the TEER value as $\leq 70 \Omega \cdot \mathrm{cm}^{2}$, to confirm opening of the junctional complex. The medium in the apical compartment was then replaced with $0.1 \mathrm{~mL}$ HBSS (with $1.8 \mathrm{mM} \mathrm{Ca}^{+2}$ ) containing PMX/DCK-OP (equivalent to $100 \mu \mathrm{g} / \mathrm{mL} \mathrm{PMX}$ ), alone or with all inhibitors except cyclosporine A, and $0.6 \mathrm{~mL}$ of HBSS (with $1.8 \mathrm{mM} \mathrm{Ca}^{+2}$ ) was added to the basolateral side of each well, followed by incubation at $37^{\circ} \mathrm{C}$ for a further $5 \mathrm{hrs}$. At predetermined time points, $0.2 \mathrm{~mL}$ of medium on the basolateral side was withdrawn and replaced with the same volume of fresh HBSS. In addition, the integrity of the monolayer was checked by measuring the TEER value, which returned to $\geq 300 \Omega \cdot \mathrm{cm}^{2}$ at 2 and $5 \mathrm{hrs}$ after drug loading, respectively, to identify restoration of the transmembrane resistance. ${ }^{28}$

All collected samples were then filtered through PVDF membrane filters $(0.45 \mu \mathrm{m})$ and the concentration of PMX that permeated across the Caco-2 cell monolayer was determined by HPLC at $254 \mathrm{~nm}$ as described above. Finally, the cumulative permeated amount of PMX from the PMX/DCK-OP was plotted as a function of time and $\mathrm{P}_{\mathrm{app}}$ was determined from the linear slope of the plot using Equation (2).

\section{In vivo pharmacokinetic study in rats}

To evaluate the beneficial effects of ion-pairing complex formation of PMX with DCK on intestinal absorption, $400 \mu \mathrm{L}$ of PMX in water $(25 \mathrm{mg} / \mathrm{kg}), \mathrm{PMX} / \mathrm{DCK}$ in water (25 mg/kg PMX), or PMX/DCK-OP (25 mg/kg PMX) was orally administered to rats. In addition, $150 \mu \mathrm{L}$ of an aqueous solution of PMX (5 mg/ $\mathrm{kg})$ was injected via the tail vein to evaluate the oral bioavailability. Next, blood samples of $150 \mu \mathrm{L}$ were collected from the retroorbital plexus at predetermined time intervals into heparinized tubes, mixed with $50 \mu \mathrm{L}$ of $3.8 \%$ sodium citrate solution, and immediately centrifuged $(2,500 \times \mathrm{g}$, 15 mins, $4{ }^{\circ} \mathrm{C}$ ). The plasma samples were separated and kept frozen at $-70{ }^{\circ} \mathrm{C}$ until analysis. Liquid chromatography/mass spectrometry (LC/MS) was performed to determine the concentration of PMX in the plasma, as described previously. ${ }^{29}$ Briefly, $300 \mu \mathrm{L}$ of $2 \% \mathrm{NH}_{4} \mathrm{OH}$ in deionized water and $100 \mu \mathrm{L}$ of standard solution were added to the plasma samples that were defrosted and centrifuged at $2,500 \times \mathrm{g}$ for $5 \mathrm{mins}$ at $4{ }^{\circ} \mathrm{C}$, followed by vortex mixing. Next, $10 \mu \mathrm{L}$ of DAMPA ( $5 \mu \mathrm{g} / \mathrm{mL}$ ) was added to each sample as an internal standard, vortex-mixed for $2 \mathrm{mins}$, and subjected to solid-phase extraction using a Plexa Bond Elut PAX cartridge (30 mg, $1 \mathrm{~mL}$; Agilent Technologies, Santa Clara, CA) in accordance with the manufacturer's instructions. The cartridges were conditioned with $500 \mu \mathrm{L}$ of methanol followed by $500 \mu \mathrm{L}$ of deionized water, and standards and samples were loaded into the cartridges. After the unadsorbed samples and impurities in the cartridges were washed with $500 \mu \mathrm{L}$ each of deionized water and methanol, elution of the adsorbed samples was performed with $2 \times 250 \mu \mathrm{L}$ of $5 \%$ formic acid prepared in methanol. The eluate was dried using a centrifugal evaporator (Genevac Ltd., Ipswich, UK), which was then reconstituted with $100 \mu \mathrm{L}$ of $5 \%$ formic acid in methanol. The concentration of PMX in the reconstituted samples was determined using an Agilent 6120 Quadruple LC/MS system with a Phenomenex Luna C18 column $(100 \times 2 \mathrm{~mm}$, $3 \mu \mathrm{m})$ as the stationary phase and acetonitrile: $0.34 \%$ formic acid $(15: 85, \mathrm{v} / \mathrm{v})$ as the mobile phase, at a flow rate of $0.2 \mathrm{~mL} / \mathrm{min}$. PMX and internal standard were ionized using an electrospray ionization (ESI) source in positive ion mode under the following source conditions: capillary voltage of $3.5 \mathrm{kV}$; drying gas flow rate of $3.1 \mathrm{~L} / \mathrm{min}$; and drying gas temperature of $300{ }^{\circ} \mathrm{C}$. Quantitative analysis of protonated molecular ions was performed at $\left([\mathrm{M}+\mathrm{H}]^{+}=428\right)$ and $\left([\mathrm{M}+\mathrm{H}]^{+}=326.1\right)$ for PMX and DAMPA, respectively.

\section{In vivo tumor growth inhibition efficacy in mice}

The antitumor effects of orally administered PMX/DCK-OP, alone or in combination with anti-PD1 administered intraperitoneally, were investigated in BALB/c mice subcutaneously inoculated with B16F10 cells at a density of $1 \times 10^{6}$ cells (in $100 \mu \mathrm{L}$ of PBS, $\mathrm{pH}$ 7.4). Mice with an average tumor volume of $40-60 \mathrm{~mm}^{3}$ were randomly divided into five groups (10 mice per group): control (untreated), PMX-IV (twice-a-week intravenous administration of $20 \mathrm{mg} / \mathrm{kg}$ PMX), Anti-PD1 (once per 3 days intraperitoneal administration of $10 \mathrm{mg} / \mathrm{kg}$ 
anti-PD1), PMX/DCK-OP (once-a-day oral administration equivalent to $20 \mathrm{mg} / \mathrm{kg} \mathrm{PMX}$ ), and combination of PMX/ DCK-OP and Anti-PD1 (once-a-day oral administration equivalent to $20 \mathrm{mg} / \mathrm{kg}$ PMX and once per 3 days intraperitoneal administration of $10 \mathrm{mg} / \mathrm{kg}$ anti-PD1). The body weight and tumor volumes of mice in all five groups were recorded every 2 days. Tumor volumes in each mouse were calculated based on the following formula: $0.52 \times \mathrm{w}^{2} \times 1$, where $\mathrm{w}$ and 1 represent the width and length of the tumor, respectively. Two weeks after oral administration, mice were sacrificed and tumor masses were measured. Induction of apoptosis in the isolated tumor tissues was detected by terminal deoxynucleotidyl transferase dUTP nick end labeling (TUNEL) assay. Moreover, microvessel expression and cell proliferation were analyzed using epithelial cell marker CD31 and proliferating cell nuclear antigen (PCNA), respectively.

\section{Pharmacokinetic and statistical analyses}

Pharmacokinetic parameters were assessed using a noncompartmental method in WinNonlin ${ }^{\circledR}$ software (ver. 5.3; Pharsight Corporation, Mountain View, CA, USA). Oneway analysis of variance (ANOVA) followed by Tukey's multiple-comparison test was used to compare more than two mean values. All data were expressed as means \pm standard deviation. In all analyses, a $P$-value less than 0.05 was considered statistically significant.

\section{Results and discussion Preparation and characterization of PMX/
DCK-OP} To enhance the lipophilicity and intestinal membrane permeability of PMX, ion-pairing complex and oral powder formulations of PMX and DCK were prepared as shown in Figure 1A. The optimal PMX/DCK-OP diluted in deionized water showed a particle size and PDI of 134 $\pm 0.098 \mathrm{~nm}$ and $0.114 \pm 0.005$, respectively, with a zeta potential of $16.3 \pm 1.00 \mathrm{mV}$. The uniformly narrow particle size and high zeta potential supports the stable formation of micelles in the aqueous phase. In addition, TEM images of PMX/DCK-OP dispersed in water confirmed the formation of spherical and homogenous nano-sized micelles with diameters $<100 \mathrm{~nm}$, which was smaller than the size observed using a DLS analyzer (Figure 1B). The

A

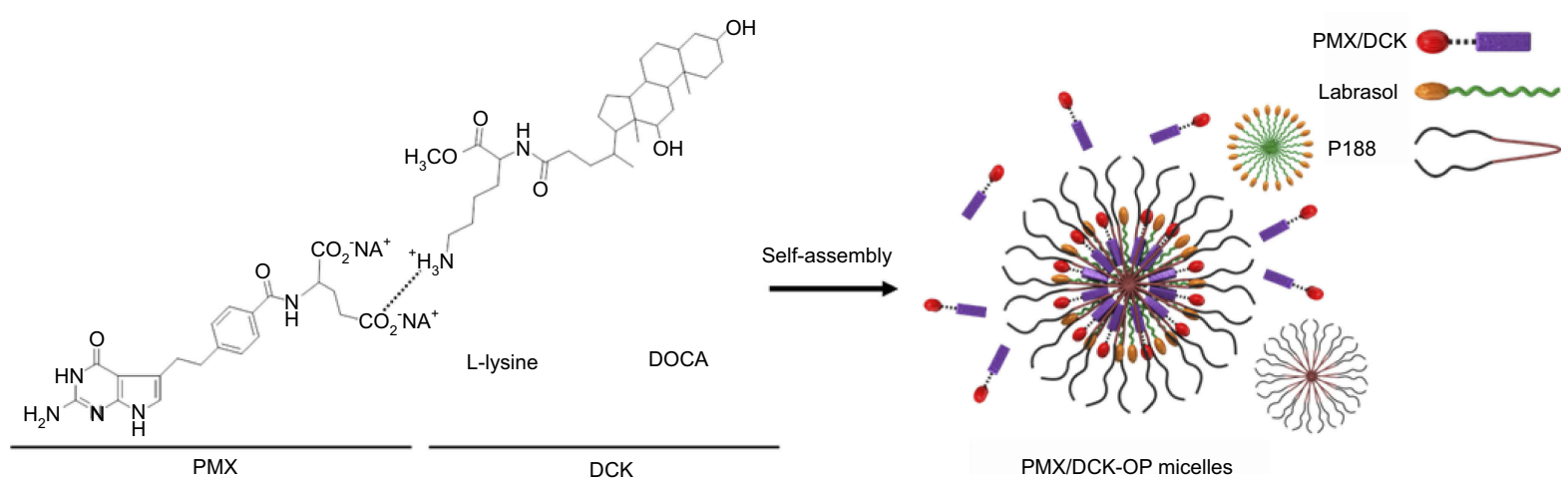

B

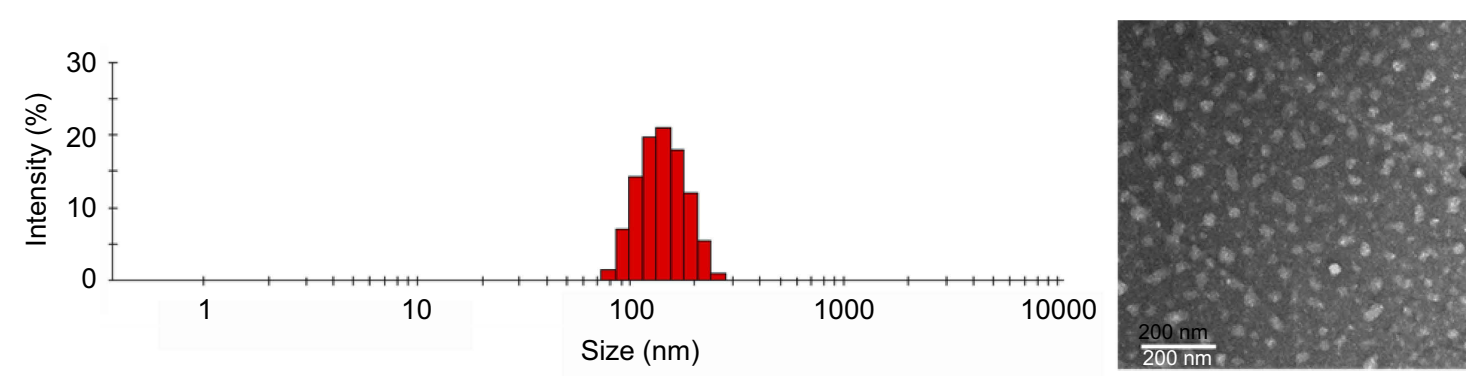

Figure I (A) Schematic illustration of ion-pairing complex formation between PMX and DCK, and self-assembled micelle formation of PMX/DCK-OP in the aqueous phase. (B) Transmission electron micrograph of PMX/DCK-OP micelles. Scale bar represents $200 \mathrm{~nm}$.

Abbreviations: PMX, pemetrexed; DCK, $\mathrm{N}^{\alpha}$-deoxycholyl-L-lysyl-methylester; PMX/DCK, ion-pairing complex between PMX and DCK; PMX/DCK-OP, oral powder formulation of PMX/DCK. 
difference in particle size of the PMX-DCK-OP micelles may be caused by the difference in sample preparation between DLS and TEM measurements. Unlike DLS, a sample for TEM is prepared in a dried state and the loosely aligned micelles can shrink during drying. The presence of Labrasol and P188 as surfactant and dispersing agent, respectively, in the optimum powder formulation were expected to increase the long-term stability and oral bioavailability of PMX. Moreover, Labrasol showed a hydrophilic-lipophilic balance (HLB) value of 14, was less toxic with high tolerance, and enhanced the intestinal absorption of hydrophilic drugs. ${ }^{30,31}$ In addition, use of $\mathrm{P} 188$ in the formulation resulted in self-assembly into micelles in the presence of aqueous phase at concentrations above the critical micellar concentration (CMC). Structurally, P188, which is an amphiphilic triblock copolymer of poly(ethylene oxide) and poly(propylene oxide), consists of a hydrophobic central core and hydrophilic outer core that can accommodate lipophilic and water soluble drugs, respectively. Furthermore, P188 has been widely used as a dispersant and solubilizing agent, and is approved for oral drug delivery. ${ }^{32,33}$

\section{In vitro permeability study of PMX/DCK-OP}

The artificial intestinal membrane permeability of PMX was significantly increased after ion-pairing complex formation with DCK and formulation of PMX/DCK-OP. The $\mathrm{P}_{\mathrm{e}}$ of PMX after ionic complex formation with DCK was increased by 36.2- and 7.29-fold compared to free PMX and PMX-OP, respectively (Table 2). The enhanced permeability of $\mathrm{PMX} / \mathrm{DCK}$ may be due to the increased lipophilicity of PMX after complex formation with the oral permeation enhancer, $\mathrm{DCK} .^{34,35}$ On the other hand, the permeability of PMX was improved 4.97-fold compared to that of free PMX after formulation with P188 and Labrasol. However, the $\mathrm{P}_{\mathrm{e}}$ of PMX/DCK-OP was increased by 3.87-, 28.2-, and 140-fold compared to
PMX/DCK, PMX-OP, and PMX, respectively. Therefore, the enhanced artificial membrane permeability of PMX/ DCK-OP could be ascribed to the synergistic action of DCK, Labrasol, and P188 in the membrane permeation of PMX. Moreover, the presence of Labrasol and P188 in the oral powder formulation may have further increased the amphiphilicity of PMX, thus facilitating passive diffusion.

The in vitro permeability data of $\mathrm{PMX}, \mathrm{PMX} / \mathrm{DCK}$, PMX-OP, and PMX/DCK-OP across Caco-2 cell monolayers are presented in Table 2. The $\mathrm{P}_{\text {app }}$ of PMX after ion-pairing complex formation with DCK was 3.35- and 1.73-fold higher than those of free PMX and PMX-OP, respectively. The improved permeability of PMX may have been due to the increased membrane flexibility and opening of transient TJs caused by binding of bile acids to the calcium channels, which enhanced paracellular drug transport. $^{36}$ Moreover, DOCA induces phosphorylation of epidermal growth factor receptor, occludin dephosphorylation, and occludin redistribution, which modulates intestinal membrane permeability. ${ }^{37}$ In addition, transcellular transport of hydrophilic drugs could be enhanced by the incorporation of bile acids into the cell membrane; this forms reverse micelles and results in the creation of aqueous channels, thereby increasing membrane permeability. ${ }^{38}$ The permeability of PMX across a Caco-2 cell monolayer was only improved 1.94-fold by adding P188 and Labrasol, while the $\mathrm{P}_{\text {app }}$ of PMX/DCK-OP was further increased by 2.46-, 4.26-, and 8.26-fold compared to those of PMX/ DCK, PMX-OP, and free PMX, respectively. This increase in the $\mathrm{P}_{\mathrm{app}}$ of PMX/DCK-OP may have been due to the formation of $\mathrm{PMX} / \mathrm{DCK}$ micelles in combination with Labrasol and P188, which could in turn increase drug absorption through endocytosis and micropinocytosis. ${ }^{39}$ In addition, nonionic surfactants, such as Labrasol and P188, can modify intestinal membrane fluidity, followed by alteration of the epithelial barrier function. ${ }^{40}$ Labrasol has been

Table 2 Effective and apparent permeability of PMX, PMX/DCK, and PMX/DCK-OP

\begin{tabular}{|c|c|c|}
\hline Test material & Effective permeability $\left(P_{e}, \times 10^{-6}, \mathrm{~cm} / \mathrm{s}\right)^{a}$ & Apparent permeability $\left(P_{\text {app }}, \times 10^{-6}, \mathrm{~cm} / \mathrm{s}\right)^{b}$ \\
\hline PMX & $0.290 \pm 0.080$ & $1.78 \pm 0.118$ \\
\hline PMX/DCK & $10.5 \pm 1.97^{* * * *}$ & $5.97 \pm 1.38^{*}$ \\
\hline PMX-OP & $1.44 \pm 0.180$ & $3.45 \pm 0.531$ \\
\hline PMX/DCK-OP & $40.6 \pm 1.48 * * * \ldots \#, \$ \$ \$$ & $14.7 \pm 3.98 * * *, \ldots \#, \$ \$ \$$ \\
\hline
\end{tabular}

Notes: Statistics: one-way ANOVA followed by Tukey's multiple-comparison test. ${ }^{a}$ Effective permeability $\left(\mathrm{P}_{\mathrm{e}}\right)$ of PMX, PMX/DCK, PMX-OP, and PMX/DCK-OP through an artificial intestinal membrane. ${ }^{b}$ Apparent permeability $\left(\mathrm{P}_{\text {app }}\right)$ of PMX, PMX/DCK, PMX-OP, and PMX/DCK-OP across a Caco-2 cell monolayer. Each value represents the mean \pm standard deviation $(n=6)$. ${ }^{*} P<0.05,{ }^{* * * P}<0.001$ compared to PMX; ${ }^{\#} P<0.001$ compared to PMX/DCK; ${ }^{\$ \$} \$<0.00 \mathrm{I}$ compared to PMX-OP.

Abbreviations: PMX, pemetrexed; DCK, $\mathrm{N}^{\alpha}$-deoxycholyl-L-lysyl-methylester; PMX/DCK, ion-pairing complex between PMX and DCK; PMX-OP, oral powder formulation of PMX; PMX/DCK-OP, oral powder formulation of PMX/DCK. 
shown to act as a permeation enhancer by disrupting TJs, causing redistribution of zonula occludens-1 (ZO-1). ${ }^{41}$ Therefore, the synergistic activity of DCK, Labrasol, and P188 may have significantly increased the $\mathrm{P}_{\mathrm{app}}$ of PMX/ DCK-OP. However, further permeability studies using gastrointestinal tissues are required to determine the stability and association constant of PMX/DCK and its oral formulation in the gastric and intestinal fluids. In addition, future studies should investigate the effects of ion-pairing complex formation with DCK and formulation with Labrasol and P188 on the intestinal permeability of PMX, and the absorption-enhancing mechanism of PMX/DCK-OP.

\section{Intestinal transport mechanism of PMX/ DCK-OP}

To determine the transport pathway involved in the active transcellular transport of PMX/DCK-OP, Caco-2 monolayers were pretreated with various biochemical inhibitors of different pathways (Figure 2). In the aqueous phase, PMX/DCK-OP may exist as PMX/DCK, which can be further incorporated into micelles together with P188 and Labrasol, as confirmed in the preparation and characterization section. The main goal of ionic complex formation of PMX with DCK was to increase the lipophilic properties and oral bioavailability of PMX via the bile acid transporter-mediated approach. The transporter specificity, affinity, and capacity are the three major factors taken into consideration in prodrug design. Bile acids are mostly reclaimed in the distal ileum by ASBT. ${ }^{42}$ Moreover, ASBT exhibits high capacity and affinity for native bile acid, suggesting that ASBT may be a useful prodrug target. ${ }^{15}$ Coupling of PMX with DCK can increase the uptake of PMX through ASBT-mediated transport. To verify the involvement of ASBT in the uptake of PMX/DCK, we inhibited ASBT using Act $\mathrm{D}$ at a concentration that had no cytotoxic effect on Caco- 2 cells; the cell viability was greater than $95 \%$ and the concentration ranged from 1 to $6 \mu \mathrm{M}$ Act $\mathrm{D}$. The cell viability at $3.2 \mu \mathrm{M}$ Act D was $105 \pm 6.81 \%$ (Figure S1). ${ }^{43}$ As a result, the $\mathrm{P}_{\text {app }}$ of $\mathrm{PMX} / \mathrm{DCK}-\mathrm{OP}$ was markedly decreased, by $75.4 \%$, compared to the control (without inhibitors) (Figure 2A). This observation was suggestive of active involvement of ASBT in the uptake of PMX/ DCK-OP. After interacting with ASBT, PMX/DCK from PMX/DCK-OP may have followed a pathway similar to that of bile acid: entry into the enterocytes; binding with intestinal bile acid-binding protein (IBABP); shuttling across the enterocytes to the basolateral membrane; and
A

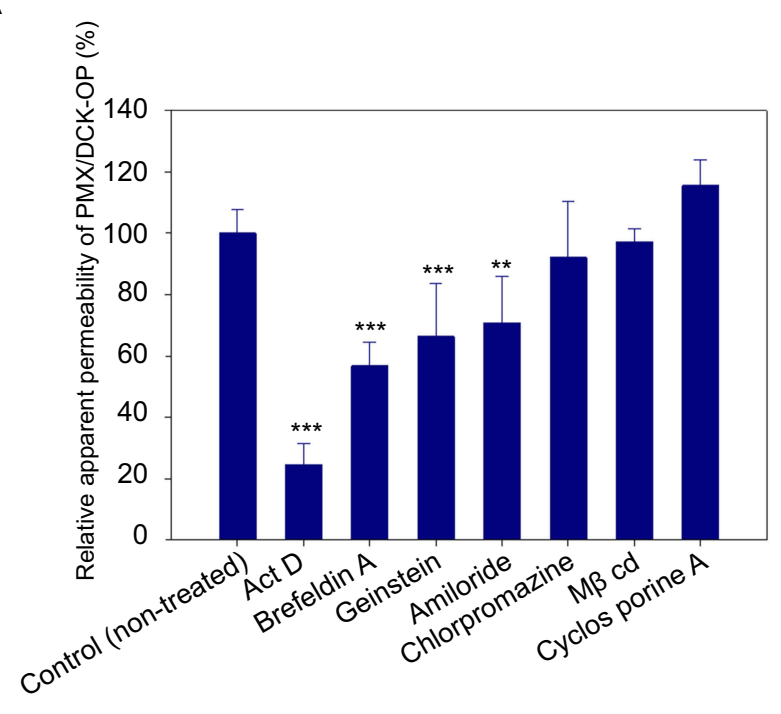

B

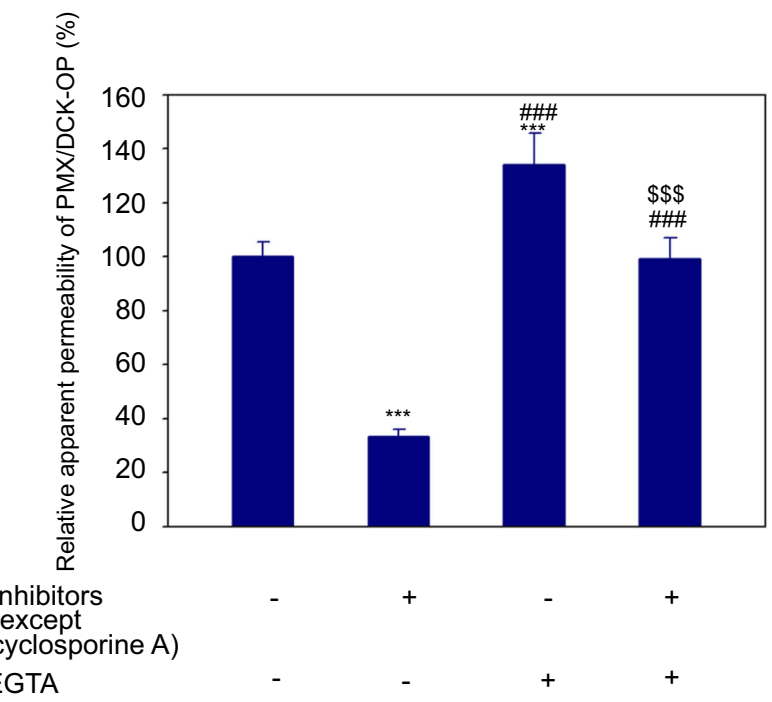

Figure 2 (A) Relative apparent permeability of PMX/DCK-OP across a Caco-2 cell monolayer after incubation with various transport inhibitors and $(\mathbf{B})$ with or without EGTA in the presence or absence of all inhibitors except cyclosporine A.

Notes: Each value represents the mean \pm standard deviation $(n=6) . * * p<0.01$, $* * * P<0.00$ I compared to the $P_{\text {app }}$ of the untreated control group. ${ }^{\#} P<0.001$ compared to the apparent permeability of PMX/DCK-OP after treatment with all inhibitors except cyclosporine $A$ in the absence of EGTA. ${ }^{\$ \$} P<0.001$ compared to the $P_{\text {app }}$ of PMX/DCKOP after treatment with EGTA in the presence of all inhibitors except cyclosporine $A$. Abbreviations: PMX, pemetrexed; DCK, $\mathrm{N}^{\alpha}$-deoxycholyl-L-lysyl-methylester; PMXI DCK, ion-pairing complex between PMX and DCK; PMX/DCK-OP, oral powder formulation of PMX/DCK; Act D, actinomycin D; M $B C D$, methyl- $\beta$-cyclodextrin; ASBT; EGTA, ethylene glycol-bis-( $\beta$-aminoethyl ether)-N,N,N',N'-tetraacetic acid; $\mathrm{P}_{\mathrm{app}}$, apparent permeability of PMX/DCK-OP across a Caco-2 cell monolayer.

effusing into the portal circulation via the recently identified heteromeric transporters, organic solute transporter alpha and beta $\left(\mathrm{OST}_{\alpha}\right.$ and $\left.\mathrm{OST}_{\beta}\right)$ (Figure 3). ${ }^{19,44}$

In addition to the ASBT-mediated transport, PMX/ DCK-OP micelles themselves can be internalized by endocytosis, because they are the major route for nanoparticle transport across the intestinal epithelium by engulfment in membrane invaginations forming intracellular vesicles. 


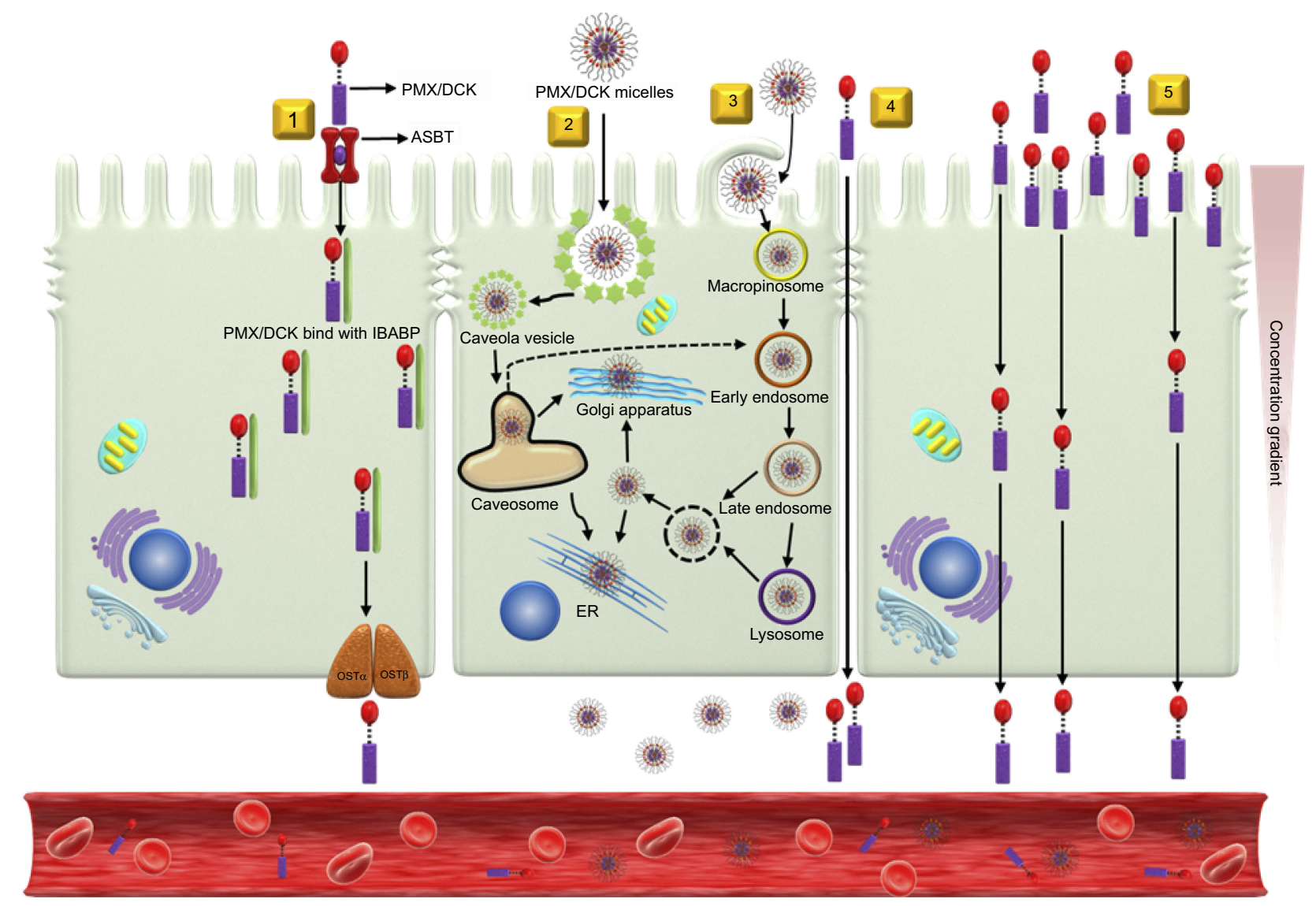

Figure 3 Schematic diagrams of possible specific and nonspecific transport pathways, and predicted intracellular trafficking and transcytosis of PMX/DCK-OP across an intestinal epithelium: (I) ASBT-mediated transport; (2) caveola/lipid raft-mediated endocytosis; (3) macropinocytosis; (4) paracellular transport; and (5) transcellular passive diffusion.

Abbreviations: PMX, pemetrexed; DCK, $\mathrm{N}^{\alpha}$-deoxycholyl-L-lysyl-methylester; PMX/DCK, ion-pairing complex between PMX and DCK; PMX/DCK-OP, oral powder formulation of PMX/DCK; ASBT, apical sodium-dependent bile acid transporter; IBABP, intestinal bile acid-binding protein; OST $_{\alpha}$, organic solute transporter alpha; OST $_{\beta}$ organic solute transporter beta; ER, endoplasmic reticulum.

First, we used inhibitors of both clathrin- and caveola/lipid raft-mediated endocytosis to explore their roles in the uptake of PMX/DCK-OP. After treatment with chlorpromazine, an inhibitor of clathrin-mediated endocytosis, the $\mathrm{P}_{\text {app }}$ of PMX/DCK-OP was only reduced by $7.95 \%$ in comparison to the control (without inhibitors), indicating the clathrin-mediated endocytosis plays a trivial role in the uptake of PMX/DCK-OP micelles (Figure 2A). On the other hand, genistein, a caveola/lipid raft-mediated endocytosis inhibitor, significantly decreased the $\mathrm{P}_{\text {app }}$ by $33.6 \%$ compared to the control (without inhibitors) (Figure 2A). Genistein is a commonly used broad-spectrum inhibitor of protein tyrosine kinase (PTK), which catalyzes tyrosine phosphorylation and activates multiple signal transduction pathways, including endocytosis regulation. ${ }^{45}$ Genistein has also been reported to be closely related with the actin network and recruitment of dynamin II, both of which are indispensable for caveola/lipid raft-mediated uptake. ${ }^{46}$ Therefore, the considerable decline in $\mathrm{P}_{\text {app }}$ in the presence of genistein confirmed the involvement of the caveola/lipid raftmediated pathway in the uptake of PMX/DCK-OP. However, there was no significant difference in the $\mathrm{P}_{\text {app }}$ after treatment of Caco-2 cells with $\mathrm{M} \beta \mathrm{CD}$, a caveola/ lipid raft-mediated endocytosis inhibitor $(2.37 \%$ decrease in $\mathrm{P}_{\mathrm{app}}$ of PMX/DCK-OP), which may be due to the difference in its mode of action. $\mathrm{M} \beta C D$ generally acts as a cholesterol-depleting agent and effectively inhibits caveola/lipid raft-mediated endocytosis. ${ }^{47}$ However, it was reported previously that the addition of cholesterol together with M $\beta C D$ may exhaust its function due to its specific interaction with cholesterol, leading to a decrease in the inhibitory efficacy of $\mathrm{M} \beta \mathrm{CD} .{ }^{27}$ Therefore, in the present study, the inhibitory activity of $M \beta C D$ may have decreased due to the presence of DOCA, a cholesterol derivative, in the DCK molecule. Macropinocytosis is another welldefined uptake mechanism that may be involved in the 
uptake of PMX/DCK-OP micelles. During this process, a large volume of fluid consisting of nanoparticles was engulfed by ruffling the plasma membrane to form cargoloaded macropinosomes. ${ }^{48}$ Here, the inhibitor of macropinocytosis, amiloride, significantly decreased the uptake of PMX/DCK-OP (by 29.0\%) compared to the control (without inhibitors), revealing the active role of macropinocytosis in the absorption of PMX/DCK-OP by the enterocytes (Figure 2A).

After entry into the cell, nanoparticles generally (i) are either captured by endosomes and consequently degraded by the lysosome, (ii) escape into the cytoplasm, or (iii) undergo recycling. In addition, nanoparticles can be localized to various organelles via endocytic vesicles and the complex intracellular trafficking machinery. ${ }^{49}$ Therefore, during the processes of endocytosis and macropinocytosis, PMX/DCK-OP micelles may be engulfed in membrane invaginations forming caveosomes and macropinosomes, and then delivered to various organelles, such as the early endosome, late endosome, and lysosome, after which they escape to the endoplasmic reticulum (ER) and Golgi apparatus, and enter the enterocytes. ${ }^{49,50}$ During this process, endosomal escape may be crucial for the intracellular delivery of PMX/DCK-OP micelles because of the digestive enzymes in the lysosome. The PMX/DCK-OP micelles escaping from the endosomes can enter the cytoplasm or exit the enterocytes via the ER and Golgi apparatus (Figure 3). However, the cytoplasmic route is usually not preferred because most drugs must enter the blood circulation to exert their pharmacological action. In addition, the nanomaterials with the required intracellular delivery density can utilize ER and Golgi routes, as well as the retrograde trafficking pathway.

It has been reported that both the Golgi complex and ER are important regulators of the secretory ER/Golgi pathway, as well as the endocytic recycling pathway. ${ }^{51}$ Therefore, to clarify whether the ER-to-Golgi apparatus is involved in the intracellular trafficking of PMX/DCKOP micelles, we examined the effects of brefeldin A, a specific inhibitor of the secretory pathway from the ER to the Golgi apparatus, ${ }^{52}$ on the permeation of PMX/DCKOP. The results indicated that the $\mathrm{P}_{\mathrm{app}}$ of PMX/DCK-OP was significantly decreased (by $43.2 \%$ ) compared to the control (without inhibitors) after treatment of the cells with brefeldin A, suggesting that the ER and Golgi apparatus are actively involved in the exocytosis of PMX/DCK-OP micelles (Figure 2A). Furthermore, the involvement of the ER-to-Golgi apparatus in the intracellular transport of
PMX/DCK-OP micelles clarified the roles of endocytosis and macropinocytosis; lysosomal escape of PMX/DCKOP micelles was also observed (Figure 3).

$\mathrm{P}$-gp is responsible for the multidrug resistance phenotype of cancer, by mediating the ATP-dependent efflux of anticancer drugs out of the cell. ${ }^{53}$ To examine the effects of P-gp-mediated efflux on the transport of PMX/DCKOP, we used cyclosporine $\mathrm{A}$ as an inhibitor of P-gp. ${ }^{54,55}$ The $\mathrm{P}_{\text {app }}$ of PMX/DCK-OP was increased by $16.0 \%$ compared to the control (without inhibitors), but the difference was not statistically significant (Figure 2A). Therefore, Pgp-mediated efflux does not have a significant influence on PMX/DCK-OP uptake.

The oral formulation can also facilitate passive diffusion and paracellular drug transport. To understand the involvement of passive diffusion and paracellular transport in the uptake of PMX/DCK-OP, we examined the effects of EGTA on the $\mathrm{P}_{\mathrm{app}}$ of PMX/DCK-OP. EGTA is a selective calcium chelator, which is used to bind free extracellular $\mathrm{Ca}^{+2}$ ions and can reversibly open the intercellular TJs of the Caco-2 monolayer. ${ }^{28}$ As shown in Figure 2B, when the Caco-2 monolayer (without EGTA) was treated with all inhibitors except cyclosporine $\mathrm{A}$, the $\mathrm{P}_{\text {app }}$ of PMX/DCK-OP decreased by $66.7 \%$ compared to the control (without inhibitors and EGTA), indicating that 33.3\% of PMX/DCK-OP was permeated by passive diffusion and paracellular transport (Figure 2B). As described above, the passive diffusion of PMX through the PAMPA membrane was markedly improved after ion-pairing complex formation with DCK, followed by formulation with Labrasol and P188. Furthermore, the $\mathrm{P}_{\text {app }}$ of PMX/DCK-OP across the EGTA-treated Caco-2 monolayer (without inhibitors) increased by $34.0 \%$ in comparison to the control (without inhibitors and EGTA), suggesting the importance of opening the TJs to increase the uptake of PMX/DCK-OP (Figure 2B). On the other hand, the $\mathrm{P}_{\text {app }}$ of EGTA-treated Caco-2 monolayer (with all inhibitors except cyclosporine A) was similar to the control (without inhibitors and EGTA) but increased by $66.0 \%$ compared to the EGTAuntreated Caco-2 monolayer (with all inhibitors except cyclosporine A). This observation also supported a significant role of EGTA in increasing the transport of PMX/ DCK-OP across the Caco-2 monolayer via reversibly opening the intracellular TJs. Therefore, it is clear that reversible opening of intracellular TJs can increase the transport of PMX/DCK-OP, and that the incorporation of components capable of opening $\mathrm{TJ}$ s in the formulation of PMX/DCK-OP can enhance the intestinal permeation of 
PMX (Figure 3). The presence of Labrasol and bile acid, which open TJs, in PMX/DCK-OP may have favored the permeation of $\mathrm{PMX} / \mathrm{DCK}-\mathrm{OP}$ through the paracellular route. $^{41,56,57}$

\section{In vivo oral absorption in rats}

The pharmacokinetic parameters in rats after intravenous administration of PMX, and oral administration of PMX, PMX/DCK, and PMX/DCK-OP, are shown in Figure 4A and $\mathrm{B}$, respectively. The maximum plasma concentration $\left(\mathrm{C}_{\max }\right)$ and area under the plasma concentration-time curve (AUC) achieved after oral administration of PMX/DCK

A

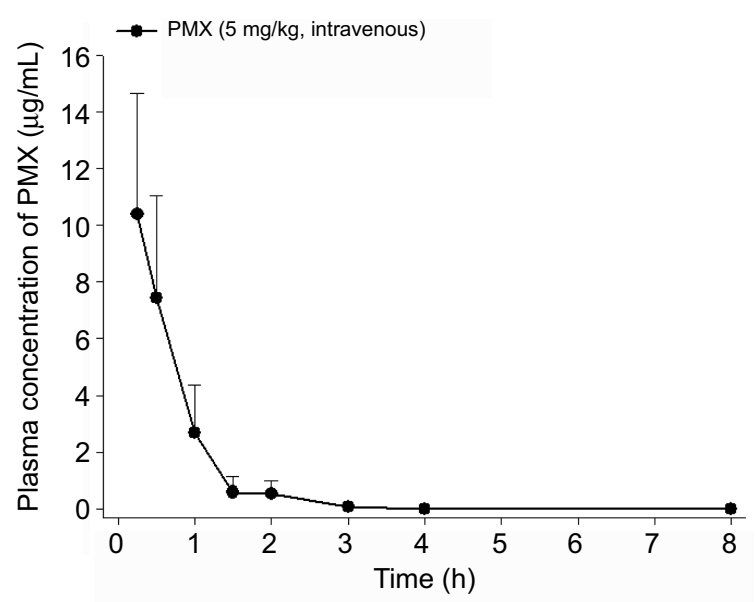

B

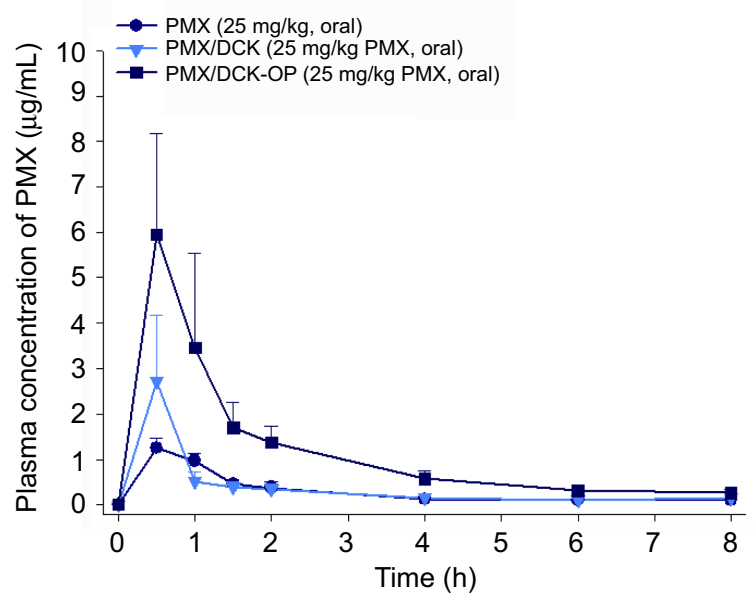

Figure 4 Venous plasma concentration-time profiles of PMX after (A) single intravenous administration of PMX $(5 \mathrm{mg} / \mathrm{kg})$ and $(\mathbf{B})$ oral administration of PMX ( $25 \mathrm{mg} / \mathrm{kg}$ ), PMX/DCK (equivalent to $25 \mathrm{mg} / \mathrm{kg}$ of PMX), or PMX/DCK-OP (equivalent to $25 \mathrm{mg} / \mathrm{kg}$ of $P M X)$ in aqueous solution to rats.

Notes: Each value represents the mean \pm standard deviation ( $n=4$ for each group). Abbreviations: PMX, pemetrexed; DCK, $\mathrm{N}^{\alpha}$-deoxycholyl-L-lysyl-methylester; PMX/DCK, ion-pairing complex between PMX and DCK; PMX/DCK-OP, oral powder formulation of PMX/DCK. (equivalent to $25 \mathrm{mg} / \mathrm{kg}$ PMX) were $4.58 \pm 4.63 \mu \mathrm{g} / \mathrm{mL}$ and $4.13 \pm 2.29 \mu \mathrm{g} \cdot \mathrm{h} / \mathrm{mL}$, respectively, which were 3.57 - and 1.76fold higher than those of oral PMX alone, suggesting significant improvement of intestinal absorption of PMX and resulting in $75.3 \%$ greater oral bioavailability of PMX/DCK than PMX (Table 3). The improvement of oral bioavailability was supported by the results of the mechanistic study, which demonstrated significant reduction of $\mathrm{P}_{\text {app }}$ after inhibition of ASBT by Act D, suggesting active involvement of bile acid transporter in the uptake of PMX/DCK. Moreover, the significant increases in in vitro artificial membrane and Caco-2 cell monolayer permeability of PMX/DCK compared to PMX confirmed the major role of paracellular and transcellular transport in the enhancement of oral bioavailability. In addition, DOCA induced disruption of intestinal barrier function by dephosphorylation of occludin, and cytoskeletal rearrangement of epithelial TJs may have enhanced the oral absorption of PMX/DCK. ${ }^{37,56-58}$ Furthermore, the $\mathrm{C}_{\max }$ and $\mathrm{AUC}_{\text {last }}$ values for PMX/DCK-OP were 1.29- and 2.25-fold higher than those of PMX/DCK, respectively. Therefore, the oral bioavailability of PMX/DCK-OP was $294 \%$ higher than that of PMX solution. The time to reach the maximum concentration $\left(\mathrm{T}_{\max }\right.$ ) was $0.63 \pm 0.25$ and $0.50 \pm 0.00 \mathrm{hrs}$ after oral administration of PMX and PMX/DCK-OP, respectively, suggesting the immediate dissolution of PMX/DCK from the powder formulation, followed by rapid absorption of PMX/DCK or PMX/DCK-OP micelles across the intestinal membrane. The enhanced oral absorption of PMX in PMX/DCK-OP proceeds as follows: (i) uptake of free PMX/DCK by the bile acid transporter, which was confirmed by inhibition of bile acid transporter; and (ii) significant decrease in $\mathrm{P}_{\text {app }}$ after inhibition of caveola/lipid raft-mediated endocytosis by genistein, suggesting internalization of PMX/DCK-OP micelles by endocytosis; 3) formation of PMX/DCK-OP macropinosomes that entered the enterocytes (which also revealed the prominent role of macropinocytosis in enhanced oral absorption); and 4) PMX/DCK-OP uptake via paracellular transport and passive diffusion, which was confirmed by reversible opening of TJs by EGTA and in vitro artificial membrane permeability.

\section{In vivo tumor growth inhibitory effect of orally administered PMX/DCK-OP}

Based on the above findings, we used the B16F10 cellbearing mouse tumor model to investigate the efficacy of combined oral administration of PMX/DCK-OP with intraperitoneal anti-PD1 in terms of inhibiting tumor progression. Compared to the untreated control group, 
Table 3 Pharmacokinetic parameters of PMX in rats after intravenous injection of PMX and oral administration of PMX, PMX/DCK, or PMX/DCK-OP

\begin{tabular}{|l|l|l|l|l|}
\hline Test material & PMX & PMX & PMX/DCK & PMX/DCK-OP \\
\hline Administration & Intravenous & Oral & Oral & Oral \\
Dose of PMX $(\mathrm{mg} / \mathrm{kg})$ & 5 & 25 & 25 & 25 \\
$\mathrm{~T}_{\max }(\mathrm{h})$ & - & $0.63 \pm 0.25$ & $0.50 \pm 0.00$ & $0.50 \pm 0.00$ \\
$\mathrm{~T}_{1 / 2}(\mathrm{~h})$ & $0.35 \pm 0.08$ & $2.50 \pm 0.45$ & $4.05 \pm 2.02$ & $2.47 \pm 0.37$ \\
$\mathrm{C}_{\max }(\mu \mathrm{g} / \mathrm{mL})$ & $15.3 \pm 6.10$ & $1.28 \pm 0.17$ & $4.58 \pm 2.63$ & $5.95 \pm 3.23$ \\
$\mathrm{AUC}$ & $9.38 \pm 4.17$ & $2.35 \pm 0.27$ & $4.13 \pm 1.29$ & $9.27 \pm 3.25^{* * * \#}$ \\
$\mathrm{AUC}$ (inf $(\mu \mathrm{g} / \mathrm{mL})$ & $9.41 \pm 4.22$ & $2.77 \pm 0.21$ & $4.90 \pm 1.73$ & $10.2 \pm 2.94^{* * * . \#}$ \\
Bioavailability $(\%)$ & & $5.02 \pm 0.57$ & $8.80 \pm 2.89$ & $19.8 \pm 6.93^{* * * \#}$ \\
\hline
\end{tabular}

Notes: Statistics: one-way ANOVA followed by Tukey's multiple-comparison test. Each value represents the mean \pm standard deviation $(\mathrm{n}=4)$. Bioavailability $(\%)$, (AUC $\mathrm{last}_{\text {, }}$

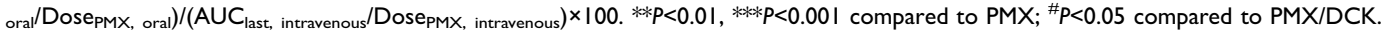

Abbreviations: PMX, pemetrexed; DCK, $\mathrm{N}^{\alpha}$-deoxycholyl-L-lysyl-methylester; PMX/DCK, ion-pairing complex between PMX and DCK; PMX/DCK-OP, oral powder formulation of PMX/DCK; $T_{\max }$, time to reach maximum plasma concentration; $T_{1 / 2}$, half-life of plasma concentration; $C_{\max }$, maximum plasma concentration; $A U C_{\text {last }}$, area under the plasma concentration-time curve from zero to the time of the last measurable plasma concentration; $\mathrm{AUC}_{\text {inf, }}$ area under the plasma concentration-time curve from zero to infinity.

the tumor growth rates were significantly delayed in mice treated with PMX/DCK-OP or PMX-IV alone (maximally suppressed by $35.5 \%$ and $38.4 \%$, respectively; Figure 5A). The improved tumor growth inhibition efficacy may have been due to the metronomic scheduling of PMX/DCK-OP and PMX-IV, which can activate both innate and adaptive immunity, induce tumor dormancy, suppress tumor angiogenesis, and render tumor cells more sensitive to T cell-mediated lysis. ${ }^{1}$ However, PMX/DCK-OP showed similar antitumor efficacy as PMX-IV, although its bioavailability was approximately $20 \%$ that of PMX-IV. This may be due to the dosing schedule of PMX/DCK-OP, which allows the drug concentration in plasma to remain constant, resulting in continuous exposure of tumor endothelial cells to the chemotherapeutic agent and allowing them to proliferate slowly, and preventing them from repairing and recovering. PMX-IV dosing (twice-weekly intravenous administration of $20 \mathrm{mg} / \mathrm{kg}$ PMX) may not be sufficient to maintain the drug concentration around the tumor tissue, resulting in a drug-free period and allowing the tumor cells to regrow and mobilize circulating endothelial progenitor cells, thus eliciting tumor neovascularization. However, further mechanistic investigations accompanied by dosing studies for metronomic PMX/DCK-OP are required to explore antitumor activity according to anti-angiogenic and immunomodulation effects. On the other hand, after intraperitoneal administration of anti-PD1, the tumor volume was significantly suppressed, by 1.35 - and 2.08 -fold, compared to the PMX/DCK-OP and control groups, respectively. In addition, the tumor volume showed a more significant reduction after combined PMX/DCK-OP and intraperitoneal anti-PD1 treatment, which resulted in maximal reduction of tumor volume by $104 \%$ and $51.5 \%$ relative to PMX/DCK-OP and Anti-PD1, respectively. This synergistic tumor growth-inhibiting activity of chemotherapy with immunotherapy may have been due to enhancement of protective $\mathrm{T}$ cell responses and the antigen-presenting function of dendritic cells. ${ }^{59}$ However, oral administration of PMX/DCK-OP or combined treatment with PMX/DCK-OP and anti-PD1 did not affect the body weight of tumor-bearing mice (Figure 5B), suggesting that the administered drugs do not have systemic toxicity. The tumors isolated on day 14 after combined treatment with oral PMX/DCK-OP and intraperitoneal anti-PD1 also showed significant decreases in tumor mass, by $69.4 \%$ and $44.2 \%$ compared to the control and oral PMX/DCK-OP groups, respectively (Figure 5C and D). Langer et al performed an open-label phase 2 cohort study using anti-PD1 (pembrolizumab) along with intravenous carboplatin and PMX, and the results showed significantly improved anticancer efficacy compared to chemotherapy alone. ${ }^{60}$ Therefore, the metronomic combination of PMX/DCKOP and intraperitoneal anti-PD1 was expected to play a synergistic role in cancer treatment. However, anticancer efficacy of intravenously administered PMX alone, or in combination with anti-PD1, must be demonstrated to confirm that the oral metronomic PMX/DCK-OP allows for a more favorable dosing schedule for synergistic anticancer effects with anti-PD1, via continuous exposure to the tumor endothelial cells, thus decreasing neoangiogenesis and boosting immune activity. 


\section{A}

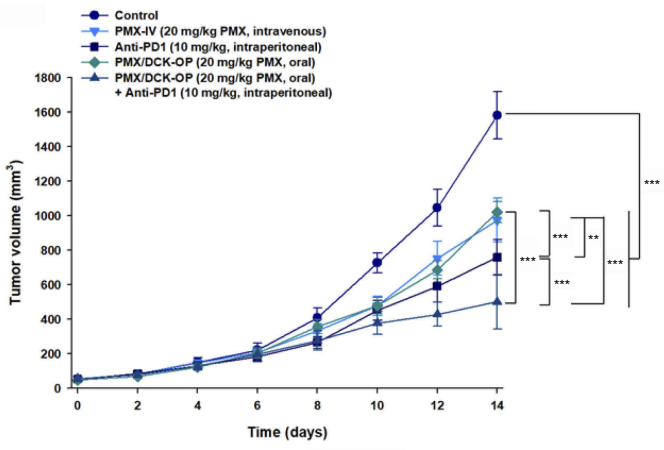

B

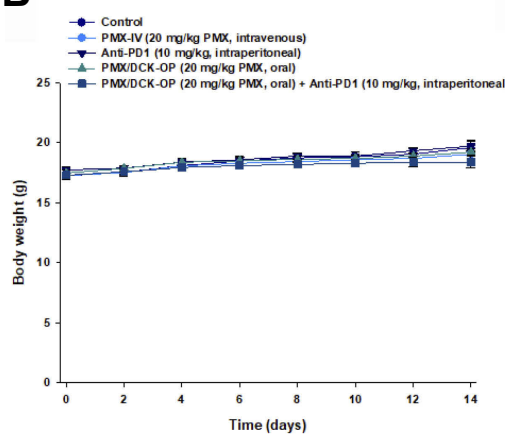

C
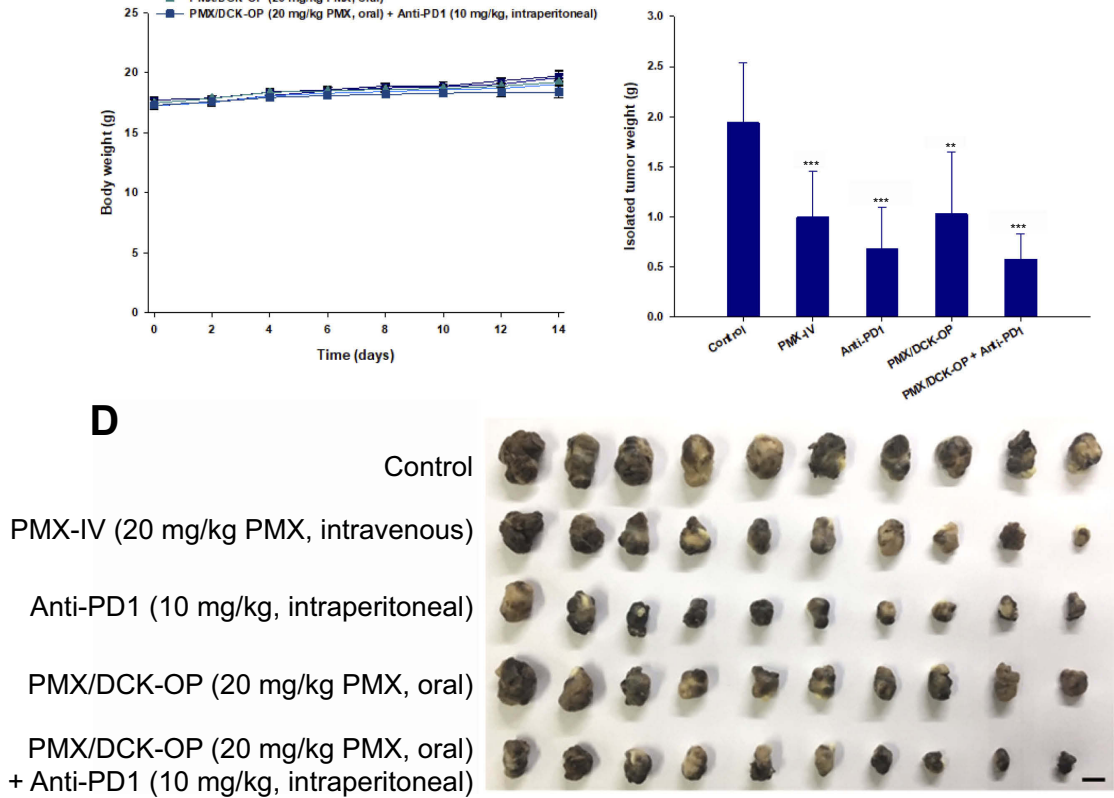

Figure 5 Efficacy of in vivo tumor growth inhibitory effects in BI6FIO tumor-bearing mice after biweekly intravenous administration of $20 \mathrm{mg} / \mathrm{kg}$ PMX (PMX-IV), intraperitoneal administration of $10 \mathrm{mg} / \mathrm{kg}$ anti-PDI (Anti-PDI) once every 3 days, once-daily oral administration of PMX/DCK-OP as $20 \mathrm{mg} / \mathrm{kg}$ PMX (PMX/DCK-OP), and combined treatment with once-daily oral administration of PMX/DCK-OP as $20 \mathrm{mg} / \mathrm{kg}$ PMX and intraperitoneal administration of $10 \mathrm{mg} / \mathrm{kg}$ anti-PDI (PMX/DCK-OP+Anti$\mathrm{PDI})$ once every 3 days for 14 days. (A) Tumor volume in mice $(* * P<0.0 \mathrm{I}, * * * \mathrm{P}<0.00 \mathrm{I})$. (B) Variation of body weight in mice during treatment. (C) Tumor weight in BI6FI0 tumor-bearing mice $(* * P<0.01$, $* * * P<0.001$ compared to the control group). (D) Photographs of tumors isolated from each group on day 15 . Scale bar represents $10 \mathrm{~mm}$. Notes: Statistics: one-way ANOVA followed by Tukey's multiple-comparison test. Each value represents the mean \pm standard deviation ( $\mathrm{n}=10$ for each group).

Abbreviations: PMX, pemetrexed; DCK, $\mathrm{N}^{\alpha}$-deoxycholyl-L-lysyl-methylester; PMX/DCK, ion-pairing complex between PMX and DCK; PMX/DCK-OP, oral powder formulation of PMX/DCK.

Histological evaluation of the tumor tissues from mice treated with PMX/DCK-OP, alone or in combination with anti-PD1 stained with PCNA, anti-CD31 antibody, and TUNEL (Figure 6) also showed similar trends. Tumors from mice treated with the combination of PMX/DCK-OP and anti-PD1 showed reduced numbers of PCNA-positive proliferative cells compared to the control and oral PMX/ DCK-OP groups. In addition, the number of CD31-positive microvessels was markedly decreased in the combined treatment group compared to the control and intravenous PMX groups. In addition, TUNEL assay showed a similar tendency, with a significantly greater number of apoptotic cells in the combined treatment group compared to the oral PMX/DCK-OP and control groups.

Based on the findings of the present study, PMX/DCKOP enhanced oral absorption, confirming the improvement in oral bioavailability of PMX with significant tumor growth suppression in combination with intraperitoneal anti-PD1 compared to PMX/DCK and anti-PD1. Therefore, PMX/DCK-OP may be suitable as a drug delivery carrier for PMX and exhibit synergistic activity with immunotherapy. 
A

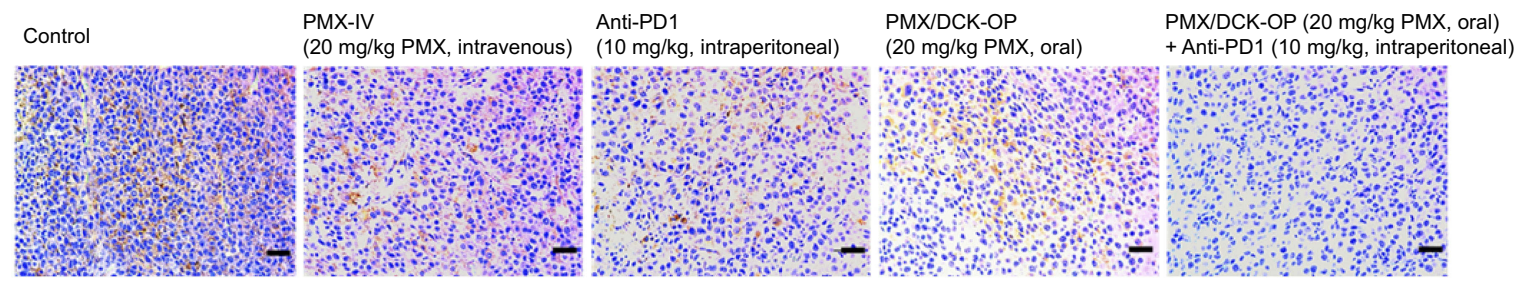

B

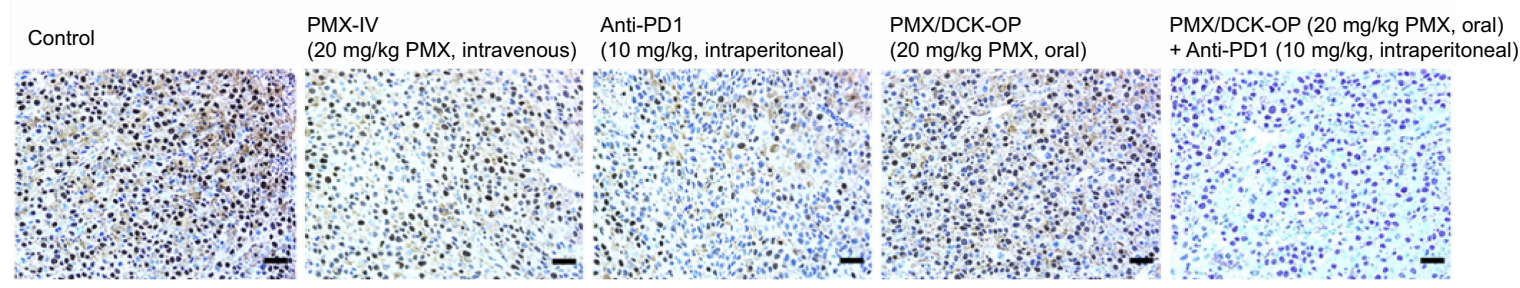

C
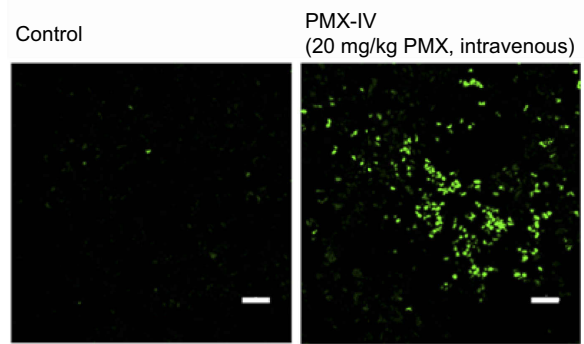

Anti-PD1

(10 mg/kg, intraperitoneal)

PMX/DCK-OP

(20 mg/kg PMX, oral)

PMX/DCK-OP (20 mg/kg PMX, oral)
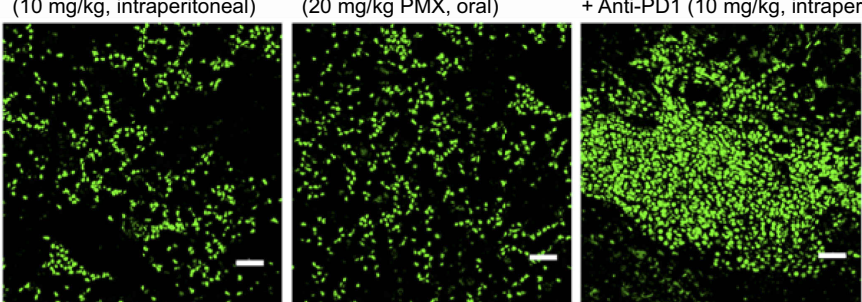

Figure 6 Representative cross-sectional images of isolated tumor tissues stained with (A) anti-CD3I antibody for microvessels (brown), (B) proliferating cell nuclear antigen (PCNA) for proliferating cells (brown), and (C) fluorescent terminal deoxynucleotidyl transferase-mediated dUPT nick end labeling (TUNEL) for apoptosis (green fluorescence) in the tumor tissues taken 14 days after treatment with various modes.

Notes: The treatment modes were biweekly intravenous administration of $20 \mathrm{mg} / \mathrm{kg}$ PMX (PMX-IV), intraperitoneal administration of $10 \mathrm{mg} / \mathrm{kg}$ anti-PDI (Anti-PDI) once every 3 days, once-daily oral administration of PMX/DCK-OP as $20 \mathrm{mg} / \mathrm{kg}$ of PMX (PMX/DCK-OP), and combined once-daily oral administration of PMX/DCK-OP as $20 \mathrm{mg} / \mathrm{kg}$ of PMX with once every 3 days intraperitoneal administration of $10 \mathrm{mg} / \mathrm{kg}$ anti-PDI (PMX/DCK-OP + Anti-PDI). Scale bars represent $50 \mu \mathrm{m}$ for anti-CD3I antibody and PCNA staining, and $20 \mu \mathrm{m}$ for TUNEL staining, respectively.

Abbreviations: PMX, pemetrexed; DCK, $\mathrm{N}^{\alpha}$-deoxycholyl-L-lysyl-methylester; PMX/DCK, ion-pairing complex between PMX and DCK; PMX/DCK-OP, oral powder formulation of PMX/DCK.

\section{Conclusion}

This study confirmed that PMX/DCK-OP enhances the intestinal membrane permeability and oral bioavailability of PMX. PMX/DCK-OP was prepared by ionic complex formation of PMX with DCK at a 1:1 molar ratio, along with Labrasol and P188 as dispersants. The optimum oral powder formulation improved the $\mathrm{P}_{\mathrm{app}}$ of PMX by 8.26-fold compared to free PMX. The results also indicated that inhibition of bile acid transporters on a Caco-2 cell monolayer significantly curtailed the $\mathrm{P}_{\text {app }}$ of PMX/DCKOP, suggesting that an ASBT-mediated pathway is the predominant mechanism underlying intestinal absorption of PMX/DCK-OP, followed by caveola/lipid raft-mediated endocytosis, macropinocytosis, passive diffusion, and paracellular transport. For these reasons, oral PMX/DCK-OP showed a $294 \%$ increase in oral bioavailability in comparison to free PMX, further confirming the role of bile acid derivative and dispersants used in the formulation. Moreover, the tumor growth was maximally suppressed after combined treatment with PMX/DCK-OP and intraperitoneal anti-PD1. These observations suggested the therapeutic potential of combined oral metronomic chemotherapy and immunotherapy for synergistic anticancer efficacy.

\section{Acknowledgment}

This study was supported by the Bio \& Medical Technology Development Program of the National Research Foundation (NRF) funded by the Korean Government Ministry of Science and ICT (MSIT; NRF-2017M3A9F5029656). 


\section{Disclosure}

The authors report no conflicts of interest in relation to this work.

\section{References}

1. Emens LA, Middleton G. The interplay of immunotherapy and chemotherapy: harnessing potential synergies. Cancer Immunol Res. 2015;3(5):436-443. doi:10.1158/2326-6066.CIR-15-0064

2. Maiti R. Metronomic chemotherapy. J Pharmacol Pharmacother. 2014;5(3):186-192. doi:10.4103/0976-500X.136098

3. Guengerich FP. Characterization of human cytochrome P450 enzymes. Faseb J. 1992;6(2):745-748. doi:10.1096/fasebj.6.11.1644261

4. Kuppens IE, Bosch TM, van Maanen MJ, et al. Oral bioavailability of docetaxel in combination with OC144-093 (ONT-093). Cancer Chemother Pharmacol. 2005;55(1):72-78. doi:10.1007/s00280-0040864-4

5. Denies S, Cicchelero L, Van Audenhove I, Sanders NN. Combination of interleukin-12 gene therapy, metronomic cyclophosphamide and DNA cancer vaccination directs all arms of the immune system towards tumor eradication. J Control Release. 2014;187:175-182. doi:10.1016/j.jconrel.2014.05.045

6. Chen CA, Ho CM, Chang MC, et al. Metronomic chemotherapy enhances antitumor effects of cancer vaccine by depleting regulatory T lymphocytes and inhibiting tumor angiogenesis. Mol Ther. 2010;18 (6):1233-1243. doi:10.1038/mt.2010.34

7. Shan F, Zhang B, Sun L, Xie L, Shen M, Ruan S. The role of combination maintenance with pemetrexed and bevacizumab for advanced stage nonsquamous non-small cell lung cancer: a systematic review and meta-analysis. Biomed Res Int. 2018;2018:5839081. doi:10.1155/2018/5839081

8. Rinaldi DA, Kuhn JG, Burris HA, et al. A phase I evaluation of multitargeted antifolate (MTA, LY231514), administered every 21 days, utilizing the modified continual reassessment method for dose escalation. Cancer Chemother Pharmacol. 1999;44(5):372-380. doi:10.1007/s002800050947

9. Hanauske AR, Chen V, Paoletti P, Niyikiza C. Pemetrexed disodium: a novel antifolate clinically active against multiple solid tumors. Oncologist. 2001;6(4):363-373. doi:10.1634/theoncologist.6-4-363

10. Thanki K, Gangwal RP, Sangamwar AT, Jain S. Oral delivery of anticancer drugs: challenges and opportunities. J Control Release. 2013;170(1):15-40. doi:10.1016/j.jconrel.2013.04.020

11. Bansal T, Akhtar N, Jaggi M, Khar RK, Talegaonkar S. Novel formulation approaches for optimising delivery of anticancer drugs based on P-glycoprotein modulation. Drug Discov Today. 2009;14 (21-22):1067-1074. doi:10.1016/j.drudis.2009.07.010

12. Aungst BJ. Absorption enhancers: applications and advances. Aaps J. 2012;14(1):10-18. doi:10.1208/s12248-011-9307-4

13. Rahman MA, Harwansh R, Mirza MA, Hussain S, Hussain A. Oral lipid based drug delivery system (LBDDS): formulation, characterization and application: a review. Curr Drug Deliv. 2011;8(4):330345. doi:10.2174/156720111795767906

14. Franke K, Kettering M, Lange K, Kaiser WA, Hilger I. The exposure of cancer cells to hyperthermia, iron oxide nanoparticles, and mitomycin $\mathrm{C}$ influences membrane multidrug resistance protein expression levels. Int J Nanomedicine. 2013;8:351-363. doi:10.2147/IJN.S37465

15. Balakrishnan A, Polli JE. Apical sodium dependent bile acid transporter (ASBT, SLC10A2): a potential prodrug target. Mol Pharm. 2006;3(3):223-230. doi:10.1021/mp060022d

16. Pangeni R, Choi JU, Panthi VK, Byun Y, Park JW. Enhanced oral absorption of pemetrexed by ion-pairing complex formation with deoxycholic acid derivative and multiple nanoemulsion formulations: preparation, characterization, and in vivo oral bioavailability and anticancer effect. Int J Nanomedicine. 2018;13:3329-3351. doi:10.2147/IJN.S177627
17. Bonina F, Puglia C, Rimoli MG, et al. Glycosyl derivatives of dopamine and L-dopa as anti-Parkinson prodrugs: synthesis, pharmacological activity and in vitro stability studies. J Drug Target. 2003;11(1):25-36.

18. Bilsky EJ, Egleton RD, Mitchell SA, et al. Enkephalin glycopeptide analogues produce analgesia with reduced dependence liability. $J$ Med Chem. 2000;43(13):2586-2590. doi:10.1021/jm000018k

19. Dawson PA, Lan T, Rao A. Bile acid transporters. J Lipid Res. 2009;50(12):2340-2357. doi:10.1194/jlr.R900012-JLR200

20. Tolle-Sander S, Lentz KA, Maeda DY, Coop A, Polli JE. Increased acyclovir oral bioavailability via a bile acid conjugate. Mol Pharm. 2004;1(1):40-48. doi:10.1021/mp034010t

21. Jeon OC, Hwang SR, Al-Hilal TA, et al. Oral delivery of ionic complex of ceftriaxone with bile acid derivative in non-human primates. Pharm Res. 2013;30(4):959-967. doi:10.1007/s11095-013-1045-0

22. Jeon OC, Seo DH, Kim HS, Byun Y, Park JW. Oral delivery of zoledronic acid by non-covalent conjugation with lysine-deoxycholic acid: in vitro characterization and in vivo anti-osteoporotic efficacy in ovariectomized rats. Eur J Pharm Sci. 2016;82:1-10. doi:10.1016/j.ejps.2015.11.004

23. Dave VS, Gupta D, Yu M, Nguyen P, Gupta SV. Current and evolving approaches for improving the oral permeability of BCS Class III or analogous molecules. Drug Dev Ind Pharm. 2017;43(2):177-189. doi:10.1080/03639045.2016.1269122

24. Park JW, Kim SJ, Kwag DS, et al. Multifunctional delivery systems for advanced oral uptake of peptide/protein drugs. Curr Pharm Des. 2015;21(22):3097-3110. doi:10.2174/1381612821666150531163944

25. Swaan PW. Recent advances in intestinal macromolecular drug delivery via receptor-mediated transport pathways. Pharm Res. 1998;15:826-834. doi:10.1023/A:1011908128045

26. Qu X, Zou Y, He C, et al. Improved intestinal absorption of paclitaxel by mixed micelles self-assembled from vitamin $\mathrm{E}$ succinate-based amphiphilic polymers and their transcellular transport mechanism and intracellular trafficking routes. Drug Deliv. 2018;25(1):210225. doi:10.1080/10717544.2018.1474967

27. He B, Lin P, Jia Z, et al. The transport mechanisms of polymer nanoparticles in Caco-2 epithelial cells. Biomaterials. 2013;34 (25):6082-6098. doi:10.1016/j.biomaterials.2013.04.053

28. Artursson P, Magnusson C. Epithelial transport of drugs in cell culture. II: effect of extracellular calcium concentration on the paracellular transport of drugs of different lipophilicities across monolayers of intestinal epithelial (Caco-2) cells. J Pharm Sci. 1990;79 (7):595-600. doi:10.1002/jps.2600790710

29. Bobin-Dubigeon C, Amiand MB, Herrenknecht C, Bard JM. Development and validation of an improved liquid chromatographymass spectrometry method for the determination of pemetrexed in human plasma. J Chromatogr B Analyt Technol Biomed Life Sci. 2009;877(24):2451-2456. doi:10.1016/j.jchromb.2009.06.020

30. Prasad YV, Puthli SP, Eaimtrakarn S, et al. Enhanced intestinal absorption of vancomycin with Labrasol and D-alpha-tocopheryl PEG 1000 succinate in rats. Int J Pharm. 2003;250(1):181-190. doi:10.1016/S0378-5173(02)00544-6

31. Kommuru TR, Gurley B, Khan MA, Reddy IK. Self-emulsifying drug delivery systems (SEDDS) of coenzyme Q10: formulation development and bioavailability assessment. Int J Pharm. 2001;212 (2):233-246. doi:10.1016/S0378-5173(00)00614-1

32. Fischer SM, Parmentier J, Buckley ST, Reimold I, Brandl M, Fricker G. Oral bioavailability of ketoprofen in suspension and solution formulations in rats: the influence of poloxamer 188. J Pharm Pharmacol. 2012;64 (11):1631-1637. doi:10.1111/j.2042-7158.2012.01541.x

33. Newa M, Bhandari KH, Li DX, et al. Preparation, characterization and in vivo evaluation of ibuprofen binary solid dispersions with poloxamer 188. Int J Pharm. 2007;343(1-2):228-237. doi:10.1016/ j.ijpharm.2007.05.031

34. Sun S, Liang N, Kawashima Y, Xia D, Cui F. Hydrophobic ion pairing of an insulin-sodium deoxycholate complex for oral delivery of insulin. Int J Nanomedicine. 2011;6:3049-3056. doi:10.2147/IJN. S25646 
35. Mrestani Y, Bretschneider B, Hartl A, Neubert RH. In-vitro and in-vivo studies of cefpirom using bile salts as absorption enhancers. J Pharm Pharmacol. 2003;55(12):1601-1606. doi:10.1211/0022357022214

36. Moghimipour E, Ameri A, Handali S. Absorption-enhancing effects of bile salts. Molecules. 2015;20(8):14451-14473. doi:10.3390/ molecules200814451

37. Raimondi F, Santoro P, Barone MV, et al. Bile acids modulate tight junction structure and barrier function of Caco-2 monolayers via EGFR activation. Am J Physiol Gastrointest Liver Physiol. 2008;294(4):G906-913. doi:10.1152/ajpgi.00043.2007

38. Pavlovic N, Golocorbin-Kon S, Ethanic M, et al. Bile acids and their derivatives as potential modifiers of drug release and pharmacokinetic profiles. Front Pharmacol. 2018;9:1-23. doi:10.3389/fphar.2018.01283

39. Sahay G, Batrakova EV, Kabanov AV. Different internalization pathways of polymeric micelles and unimers and their effects on vesicular transport. Bioconjug Chem. 2008;19(10):2023-2029. doi:10.1021/ bc8002315

40. Gupta S, Kesarla R, Omri A. Formulation strategies to improve the bioavailability of poorly absorbed drugs with special emphasis on self-emulsifying systems. ISRN Pharm. 2013;2013:1-16.

41. DiMarco RL, Hunt DR, Dewi RE, Heilshorn SC. Improvement of paracellular transport in the Caco-2 drug screening model using protein-engineered substrates. Biomaterials. 2017;129:152-162. doi:10.1016/j.biomaterials.2017.03.023

42. Shneider BL. Intestinal bile acid transport: biology, physiology, and pathophysiology. J Pediatr Gastroenterol Nutr. 2001;32(4):407-417. doi:10.1097/00005176-200104000-00002

43. Kanda T, Foucand L, Nakamura Y, et al. Regulation of expression of human intestinal bile acid-binding protein in Caco-2 cells. Biochem J. 1998;330(Pt 1):261-265. doi:10.1042/bj3300261

44. Dawson PA, Karpen SJ. Intestinal transport and metabolism of bile acids. J Lipid Res. 2015;56(6):1085-1099. doi:10.1194/jlr.R054114

45. Hubbard SR, Till JH. Protein tyrosine kinase structure and function. Annu Rev Biochem. 2000;69:373-398. doi:10.1146/annurev. biochem.69.1.373

46. Orlandi PA, Fishman PH. Filipin-dependent inhibition of cholera toxin: evidence for toxin internalization and activation through caveolae-like domains. J Cell Biol. 1998;141(4):905-915. doi:10.1083/jcb.141.4.905

47. Dutta D, Donaldson JG. Search for inhibitors of endocytosis: intended specificity and unintended consequences. Cell Logist. 2012;2(4):203-208. doi:10.4161/cl.23967

48. Kerr MC, Teasdale RD. Defining macropinocytosis. Traffic. 2009;10 (4):364-371. doi:10.1111/tra.2009.10.issue-4
49. Azevedo C, Macedo MH, Sarmento B. Strategies for the enhanced intracellular delivery of nanomaterials. Drug Discov Today. 2018;23 (5):944-959. doi:10.1016/j.drudis.2017.08.011

50. Singh S, Kumar A, Karakoti A, Seal S, Self WT. Unveiling the mechanism of uptake and sub-cellular distribution of cerium oxide nanoparticles. $\mathrm{Mol}$ Biosyst. 2010;6(10):1813-1820. doi:10.1039/c0mb00014k

51. Burd CG. Physiology and pathology of endosome-to-Golgi retrograde sorting. Traffic. 2011;12(8):948-955. doi:10.1111/j.16000854.2011.01188.x

52. Klausner RD, Donaldson JG, Lippincott-Schwartz J. Brefeldin A: insights into the control of membrane traffic and organelle structure. $J$ Cell Biol. 1992;116(5):1071-1080. doi:10.1083/jcb.116.5.1071

53. Lee SD, Osei-Twum JA, Wasan KM. Dose-dependent targeted suppression of P-glycoprotein expression and function in Caco-2 cells. Mol Pharm. 2013;10(6):2323-2330. doi:10.1021/mp300668e

54. Tang F, Horie K, Borchardt RT. Are MDCK cells transfected with the human MRP2 gene a good model of the human intestinal mucosa? Pharm Res. 2002;19(6):773-779. doi:10.1023/A:1016192413308

55. Hu K, Cao S, Hu F, Feng J. Enhanced oral bioavailability of docetaxel by lecithin nanoparticles: preparation, in vitro, and in vivo evaluation. Int J Nanomedicine. 2012;7:3537-3545. doi:10.2147/IJN. S30631

56. Forsgard RA, Korpela R, Stenman LK, Osterlund P, Holma R. Deoxycholic acid induced changes in electrophysiological parameters and macromolecular permeability in murine small intestine with and without functional enteric nervous system plexuses. Neurogastroenterol Motil. 2014;26(8):1179-1187. doi:10.1111/nmo.12383

57. Mithani SD, Bakatselou V, TenHoor CN, Dressman JB. Estimation of the increase in solubility of drugs as a function of bile salt concentration. Pharm Res. 1996;13(1):163-167. doi:10.1023/A:1016062224568

58. Tian C, Asghar S, Wu Y, et al. Improving intestinal absorption and oral bioavailability of curcumin via taurocholic acid-modified nanostructured lipid carriers. Int J Nanomedicine. 2017;12:7897-7911. doi:10.2147/IJN.S145988

59. Chen YL, Chang MC, Cheng WF. Metronomic chemotherapy and immunotherapy in cancer treatment. Cancer Lett. 2017;400:282-292. doi:10.1016/j.canlet.2017.01.040

60. Langer CJ, Gadgeel SM, Borghaei H, et al. Carboplatin and pemetrexed with or without pembrolizumab for advanced, non-squamous non-small-cell lung cancer: a randomised, phase 2 cohort of the openlabel KEYNOTE-021 study. Lancet Oncol. 2016;17(11):1497-1508. doi:10.1016/S1470-2045(16)30498-3 


\section{Supplementary material}

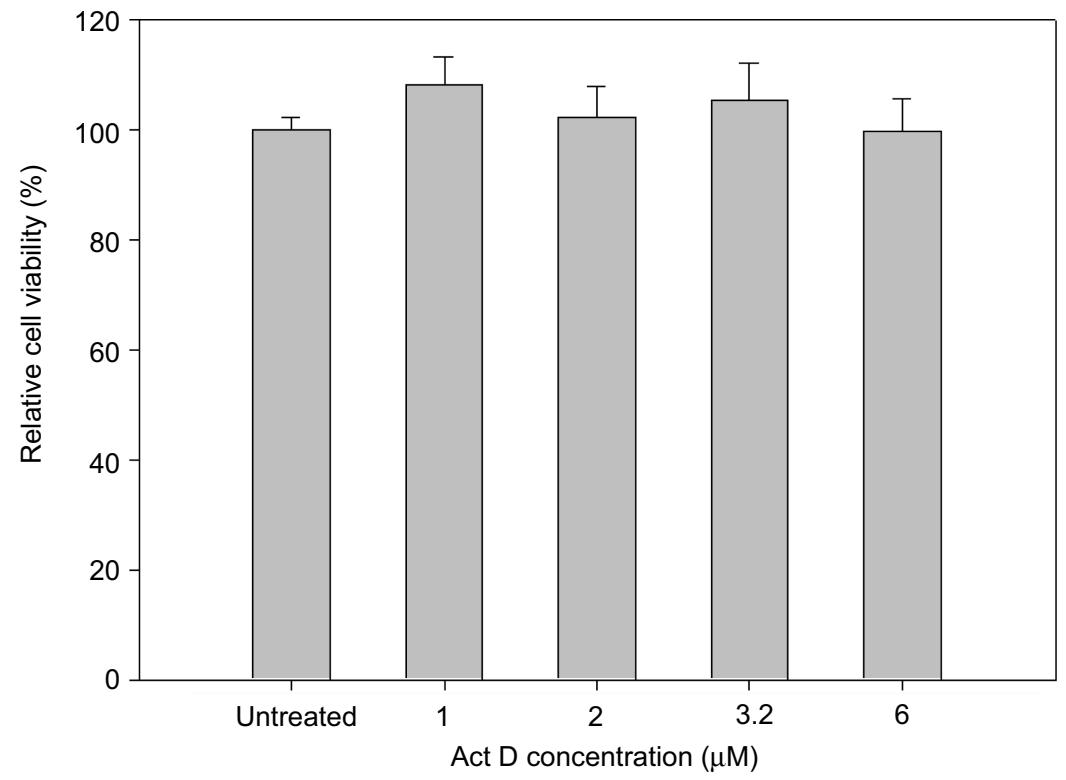

Figure SI In vitro cytotoxic effect of Act D on Caco-2 cells.

Notes: Cell viability was measured using WST-8 and the growth of Caco-2 cells compared to the untreated control group. All data are expressed as means \pm standard deviation $(n=6)$

Abbreviation: Act D, actinomycin D.

\section{Publish your work in this journal}

The International Journal of Nanomedicine is an international, peerreviewed journal focusing on the application of nanotechnology in diagnostics, therapeutics, and drug delivery systems throughout the biomedical field. This journal is indexed on PubMed Central, MedLine, CAS, SciSearch ${ }^{\mathbb{R}}$, Current Contents ${ }^{\mathbb{R}} /$ Clinical Medicine,
Journal Citation Reports/Science Edition, EMBase, Scopus and the Elsevier Bibliographic databases. The manuscript management system is completely online and includes a very quick and fair peer-review system, which is all easy to use. Visit http://www.dovepress.com/ testimonials.php to read real quotes from published authors. 